\title{
Revisiting the population of Galactic open clusters
}

\author{
A. E. Piskunov ${ }^{1,2,3}$, N. V. Kharchenko ${ }^{1,2,4}$, S. Röser ${ }^{2}$, E. Schilbach ${ }^{2}$, and R.-D. Scholz ${ }^{1}$
}

\author{
${ }^{1}$ Astrophysikalisches Institut Potsdam, An der Sternwarte 16, 14482 Potsdam, Germany \\ e-mail: [apiskunov;nkharchenko;rdscholz]@aip.de \\ 2 Astronomisches Rechen-Institut, Mönchhofstraße 12-14, 69120 Heidelberg, Germany \\ e-mail: [apiskunov; elena;nkhar;roeser]@ari.uni-heidelberg.de \\ 3 Institute of Astronomy of the Russian Acad. Sci., 48 Pyatnitskaya Str., 109017 Moscow, Russia \\ e-mail: piskunov@inasan.rssi.ru \\ 4 Main Astronomical Observatory, 27 Academica Zabolotnogo Str., 03680 Kiev, Ukraine \\ e-mail: nkhar@mao.kiev.ua
}

Received 5 July 2005/ Accepted 22 August 2005

\section{ABSTRACT}

We present results of a study of the galactic open cluster population based on the all-sky catalogue ASCC-2.5 (I/280A) compiled from Tycho-2, Hipparcos and other catalogues. The sample of optical clusters from ASCC- 2.5 is complete up to about 850 pc from the Sun. The symmetry plane of the clusters' distribution is determined to be at $Z_{0}=-22 \pm 4 \mathrm{pc}$, and the scale height of open clusters is only $56 \pm 3 \mathrm{pc}$. The total surface density and volume density in the symmetry plane are $\Sigma=114 \mathrm{kpc}^{-2}$ and $D\left(Z_{0}\right)=1015 \mathrm{kpc}^{-3}$, respectively. We find the total number of open clusters in the Galactic disk to be of order of $10^{5}$ at present. Fluctuations in the spatial and velocity distributions are attributed to the existence of four open cluster complexes (OCCs) of different ages containing up to a few tens of clusters. Members in an OCC show the same kinematic behaviour, and a narrow age spread. We find, that the youngest cluster complex, OCC $1(\log t<7.9)$, with 19 deg inclination to the Galactic plane, is apparently a signature of Gould's Belt. The most abundant OCC 2 complex has moderate age (log $t \approx 8.45$ ). The clusters of the Perseus-Auriga group, having the same age as OCC 2, but different kinematics are seen in breaks between Perseus-Auriga clouds. The oldest ( $\log t \approx 8.85$ ) and sparsest group was identified due to a large motion in the Galactic anticentre direction. Formation rate and lifetime of open clusters are found to be $0.23 \pm 0.03 \mathrm{kpc}^{-2} \mathrm{Myr}^{-1}$ and $322 \pm 31 \mathrm{Myr}$, respectively. This implies a total number of cluster generations in the history of the Galaxy between 30 to 40 . We estimate that less than about $10 \%$ of the total Galactic stellar disk population has ever passed an open cluster membership.

Key words. Galaxy: disk - Galaxy: kinematics and dynamics - open clusters and associations: general - solar neighbourhood Galaxy: stellar content - Galaxy: structure

\section{Introduction}

Open clusters play a double role in astrophysical studies. Since they consist of stars which have been born and lived together, they provide natural laboratories to prove or rule out theories of the formation and evolution of stars. Due to the fact that all members have about the same distance from the observers, that they show common space motion, and have uniform chemical composition, each single cluster represents excellent empirical reference sequences which are basic for many contemporary scales and calibrations used in astrophysics.

On the other hand, studying the open cluster population provides important information that contributes to a better understanding of the structure and evolution of the Milky Way. In principal, basic parameters like distance, motion, age, and metallicity can be determined for an open cluster more accurately than for a single field star (roughly by a factor of $\sqrt{n}$ where $n$ is the number of cluster members). Actually, they are better tracers of large scale structures of the Galactic disk population than field stars. The most comprehensive studies of the Galactic cluster population are about 20 years old (Lyngå 1982; Janes et al. 1988). They were based on the best data available at that time, the Lund Catalogue of Open Cluster Data (Lyngå 1987, hereafter, the Lund Catalogue) and its subset of clusters with 3-colour photometry (Janes \& Adler 1982). Although these studies present an important step in our understanding of the general properties of the cluster population, they suffer from incompleteness of the cluster samples and from inhomogeneity of the clusters parameters.

This can be illustrated by the following statistics. About 1200 clusters were known in the Lund catalogue by 1988. Only 400 of them had accurate, but heterogeneous $U B V$ photometry, and photometric distances, reddening and age values. Although for almost all clusters apparent diameters were given in the Lund catalogue (estimated by eye from sky charts or defined by the size of detector's field of 
view), only about 100 clusters were studied in a systematic way by use of stellar counts (Danilov \& Seleznev 1993). Kinematics, being the most traditional field in open cluster studies, was also hampered by heterogeneous proper motions and radial velocities (RVs), and less than 100 clusters had proper motions reduced to the fundamental reference system (van Schewick 1971). Space velocities were available for a few tens of clusters only. Almost nothing was known about characteristic values of cluster statistics, how complete and representative the sample was, i.e. the basic parameters needed for a systematic study of typical properties of the Galactic cluster population. These difficulties were mainly due to the fact that data on various clusters were strongly inhomogeneous since they were obtained by different instruments, detectors and techniques, with little effort to reduce them into one single system.

Since that time considerable improvement has been achieved in the field of new and homogeneous observations which could be used for the determination of basic cluster parameters and for a systematic search for new clusters. First of all, there are the accurate all-sky surveys like Hipparcos/Tycho in the optical and 2MASS in the infrared. The first efforts to look for open clusters in these data led to the discovery of new objects both located in the Solar neighbourhood, and at large distances from the Sun. Bica et al. (2003a) compiled a list of 276 embedded infrared star clusters and stellar groups from the literature and were able to increase the number by 346 new compact and remote objects associated with nebulae by making use of the 2MASS data (Dutra et al. 2003; Bica et al. 2003b). Also, nearby clusters were discovered in the Hipparcos/Tycho catalogues. Platais et al. (1998) found 15 nearby, very loose and extended clusters and associations in the Hipparcos catalogue, whereas Alessi et al. (2003) discovered 11 new clusters within $0.8 \mathrm{kpc}$ with the Tycho data.

However, with respect to the astrophysical parameters for clusters, the situation had not much improved during recent years: the parameter set is neither complete nor homogeneous. The on-line list of open cluster data by Dias et al. (2004, DLAM hereafter, see also Dias et al. 2002) which can be considered as a continuation of the Lund Catalogue now contains by a factor of 1.5 more clusters than its predecessor, but the degree of completeness of this list is still unknown. Since the cluster data in the DLAM list are taken from the literature (or from private communications), the sets of the derived parameters differ from cluster to cluster. Also, the parameters themselves are based on heterogeneous observations and different methods of cluster definition and of parameter determination. Whenever using these data for cluster population studies, one would meet problems caused by uncertain cluster statistics and data heterogeneity. Even one of the most uniform lists of cluster parameters, the Loktin et al. (2001, 2004) catalogue, is based on non-uniform photometric data and uncertain membership. In other words, for studies of the general properties of the Galactic cluster population, we cannot simply benefit from a larger sample of open clusters as long as we cannot estimate its homogeneity.

Ideal preconditions for cluster population studies are deep and uniform sky surveys of sufficiently accurate astrometric and photometric data supplemented by radial velocities, metallicities, and spectral classifications. Statistical properties of a sample of clusters identified with these data could be well estimated since its completeness should correlate with the completeness limit of the surveys used. Applying uniform criteria and methods of membership and parameter determination, one would get a homogeneous set of basic cluster parameters. In the near future an important step can be undertaken in this direction when the 2 MASS data will be combined with the final version of the UCAC catalogue, and with the RAVE and SEGUE surveys. In the further future, the observations of the GAIA satellite will provide data at an even higher level of accuracy and homogeneity.

At the moment, more or less complete and accurate data are available only for relatively bright stars and consequently for relatively nearby clusters. Nevertheless, it is worth trying to use the present data for the re-determination of cluster memberships and for the re-estimation of basic cluster parameters by applying uniform methods to homogeneous date sets. This gives us a basis to increase the reliability of the results on general properties of clusters, to estimate the possibilities and constraints of the data available, and therefore, to update our knowledge on the Galactic cluster population, now.

We started a long trip from the available observations to study the population of Galactic open clusters with the compilation of a complete survey of stars and with the reduction (if necessary) of the data to common reference systems. This effort resulted in the All-Sky Compiled Catalogue of 2.5 million stars (ASCC-2.5 ${ }^{1}$, Kharchenko 2001) with absolute proper motions in the Hipparcos system, with $B, V$ magnitudes in the Johnson photometric system, and supplemented with spectral types and radial velocities if available. The ASCC-2.5 was used to identify known open clusters and compact associations from the Lund Catalogue, the Dias et al. (2004) on-line data collection, and the Ruprecht et al. (1981) list of associations. In the ASCC-2.5 we found 520 of about 1700 known clusters (Kharchenko et al. 2004b, hereafter Paper I) and discovered 130 new open clusters (Kharchenko et al. 2005b, hereafter Paper III). A pipeline was developed to determine cluster membership based on kinematic and photometric criteria as well as to obtain a uniform set of cluster structural, kinematic and evolutionary parameters (see Paper I and Kharchenko et al. 2005a, hereafter Paper II).

In this paper we use the results of Papers II and III to study unbiased properties of the cluster population of the local Galactic disk. In Sect. 2 we briefly describe the data set. The completeness of the cluster sample and the spatial distribution of open clusters in the Galactic plane and perpendicular to it is discussed in Sect. 3. The kinematics of the system of open clusters is considered in Sect. 4. Significant irregularity in the spatial and kinematic distributions of clusters revealed complexes and groups of clusters which are discussed in Sect. 5. In Sect. 6 we determine typical lifetime and formation rate of clusters as it follows from the present statistics. Concluding remarks are given in Sect. 7.

\footnotetext{
$1 \mathrm{ftp}: / /$ cdsarc.u-strasbg.fr/pub/cats/I/280A
} 


\section{Summary of the data used}

The present sample contains 520 already known clusters which we identified in the ASCC-2.5 (Paper I) and 130 new open clusters which we newly detected in the ASCC-2.5 data (Paper III). This sample of 650 open clusters is homogeneous in many aspects: it is based on a practically uniform data set (i.e. the catalogue ASCC-2.5 with proper motions and magnitudes reduced to uniform astrometric and photometric systems), on a uniform approach of membership selection (kinematic and photometric criteria, see Paper I) and on a uniform technique of the determination of cluster parameters applied in Papers II and III. For each cluster in our sample, we determined distance and celestial position (i.e. the location in 3D-space), reddening, size (including core and corona radii), kinematics (the mean proper motions in the Hipparcos system), and the cluster age computed from ages of individual members near the Main Sequence turn-off. On the basis of a new cluster membership determination, we derived radial velocities and spatial velocity vectors for 359 clusters of our sample. For details of the above parameter determination we refer the reader to Paper II. The results are presented in two data sets: the Catalogue of Open Cluster Data and its Extension 1. The catalogues are only available in electronic form at the CDS online archive ${ }^{2}$. Hereafter, we refer to both catalogues with the same identifier COCD.

The two closest clusters, the Hyades and Collinder 285 (the UMa cluster), are missing in our list. Due to their proximity to the Sun, they occupy large areas on the sky and require a specific technique of membership determination. In order to complete our sample, we have taken the corresponding data for these clusters from Loktin et al. (2004) and the WEBDA ${ }^{3}$ database. The mean proper motions and radial velocities for both clusters were computed with data of the ASCC-2.5 and CRVAD $^{4}$ (Kharchenko et al. 2004a) catalogues. All our discussions in the following therefore refer to this sample of 652 clusters.

Although the current sample contains clusters covering a wide range of ages (from $\log t=6.6 \ldots 9.5$ ), it is strongly biased towards younger ages. This is not surprising for an unpreselected cluster sample: due to dynamical evolution and evaporation of members, the typical life time of a cluster is of the order of a few hundred Myr. On the other hand, our cluster sample is magnitude limited. Since young clusters contain, in general, more luminous stars, they dominate at larger distances and their proportion in the sample is somewhat higher than that of older clusters. Thus, in average our sample represents the moderately young disk.

\section{Spatial distribution in the disk}

Throughout the paper we use the rectangular coordinate system $X, Y, Z$ with origin in the barycentre of the Solar system,

\footnotetext{
2 ftp://cdsarc.u-strasbg.fr/pub/cats,

http://vizier.u-strasbg.fr

3 http://obswww. unige.ch/webda/

4 Catalogue of Radial Velocities with Astrometric Data can be retrieved from the CDS by

ftp: //cdsarc.u-strasbg.fr/pub/cats/III/239
}

and axes pointing to the Galactic centre $(X)$, to the direction of Galactic rotation $(Y)$, and to the North Galactic pole $(Z)$. The corresponding components of the space velocity are $U, V, W$, respectively. Unless other specifications are given, we consider the $(X, Y)$ plane to be the Galactic plane.

\subsection{The completeness of the sample and the local density enhancement}

In order to estimate the spatial completeness of the sample, we studied the distribution of clusters in the $(X, Y)$ plane and computed their surface density $\Sigma$ as a function of the projected distance $d_{x y}$ from the Sun. The corresponding histogram is shown in the left panel of Fig. 1. Due to the low number of clusters at small distances from the Sun, the first bin represents a circle with a radius of $150 \mathrm{pc}$. All other bins are concentric rings of $100 \mathrm{pc}$ width each. With the exception of a considerable excess at $d_{x y}=0.35 \ldots 0.55 \mathrm{kpc}$, the distribution is almost flat up to $d_{x y}=0.85 \mathrm{kpc}$. At larger distances, the surface density $\Sigma$ of clusters is steadily decreasing. Assuming a uniform density model for the distribution of clusters in the Solar neighbourhood, we can interpret this behaviour as evidence for an increasing incompleteness of our sample at projected distances larger than $0.85 \mathrm{kpc}$. We adopt $d_{x y}^{c}=0.85 \mathrm{kpc}$, as the completeness limit of our sample.

On the other hand, the enhancement of the observed cluster density at $d_{x y}=0.35 \ldots 0.55 \mathrm{kpc}$ is too significant to be attributed to random fluctuations in the spatial distribution of open clusters in the Solar neighbourhood. Assuming a density of $100 \pm 10$ clusters per square kpc as typical of the "cluster field" around the Sun, we would expect $56 \pm 8$ clusters at $d_{x y}=0.35 \ldots 0.55 \mathrm{kpc}$. Instead of this, 91 clusters are counted in this region. Does this enhancement describe a general property of the local galactic structure or is this excess caused by a particular group (groups) of open clusters with a common evolutionary history?

In order to understand the nature of this feature, we construct the surface density distribution for samples of different ages. Altogether four cluster generations are considered: the sample T1 including the 269 youngest clusters with ages $\log t \leq 7.9$; T2 with 101 moderately young clusters of ages of $\log t=(7.9,8.3]$; T3 with 132 intermediate age clusters of $\log t=(8.3,8.6]$; T4 with 150 older clusters, $\log t>8.6$. The age ranges are selected in such a way that the pattern of density distributions reveals the highest contrast. For each sample we construct the histogram of the surface density, smoothed with a five-point rectangular filter. The bin size is selected to be $0.025 \mathrm{kpc}$.

The results are shown in the right panels of Fig. 1. The horizontal dashed lines are average levels of the "field" surface density $\Sigma_{0, i}$, determined separately for each sample. For the samples T2 and T4, they are simple averages of the densities within the completeness area. The first bins in both groups, where by chance one single nearby cluster produces a maximum of the distribution, are not taken into account due to their low statistical significance. For the samples T1 and T3 the "field" density is estimated from different ranges of distance: $\Sigma_{0,1}$ is computed 

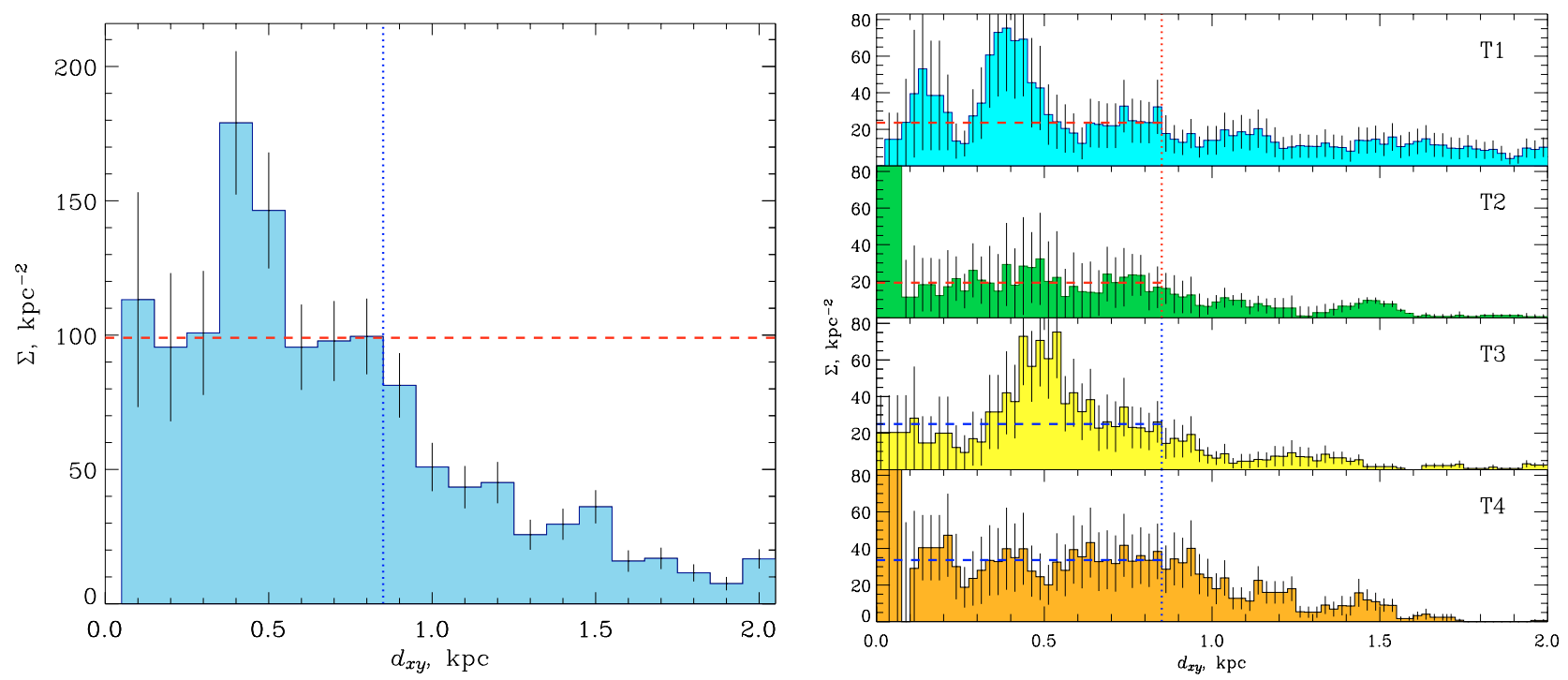

Fig. 1. Distribution of the surface density $\Sigma$ of open clusters versus their distance $d_{x y}$ from the Sun projected onto the Galactic plane. The dotted lines indicate the completeness limit, the dashed horizontal lines correspond to the average density of "field clusters" (i.e., not contained in cluster complexes, s. Sect. 5). The bars are Poisson errors derived from cluster counts. Left panel: distribution of all clusters. Right panels: distributions of clusters with different ages. The panel designations from $\mathrm{T} 1$ to $\mathrm{T} 4$ are the identifiers of four subsamples of different ages as defined in the text.

from data in $d_{x y}=0.50 \ldots 0.85 \mathrm{kpc}$, whereas for $\Sigma_{0,3}$ we consider a distance range of $d_{x y}=0.65 \ldots 0.85 \mathrm{kpc}$. We discuss these groups in more detail in Sect. 5.

\subsection{Open clusters and interstellar extinction}

Studies of the distribution of the obscuring matter in the Galactic disk have been carried out for many years (Sharov 1963; FitzGerald 1968; Lucke 1978; Neckel \& Klare 1980; Arenou et al. 1992). Usually, the methods make use of photometric data and spectral classifications of field stars over the whole sky and provide both reddening and distance evaluation. As a rule, these observations allow a mapping of interstellar extinction within the local disk, typically up to $3 \mathrm{kpc}$. Recently, Hipparcos parallaxes and data on the equivalent widths of $\mathrm{NaI}$ lines in spectra of about 1000 local stars were used for a detailed mapping of the immediate $(d<0.3 \mathrm{kpc})$ Solar neighbourhood (Lallement et al. 2003). As an upper limit of extinction the maps constructed in Schlegel et al. (1998) can be taken, although they are of limited use in the $1 \mathrm{kpc}$ neighbourhood of the Sun.

Since open clusters reside in the Galactic disk, one must expect that they are subject of sometimes strong and irregular interstellar extinction which should be taken into account in their study. On the other hand, open clusters having a compact structure, accurate distances, and with reddening determined, as a rule, from a number of stars could be used as suitable probes tracing the true distribution of reddening in the Galactic disk. The purpose of this section is to reveal the important features of the distribution of interstellar extinction from data on reddening of open clusters.

In Fig. 2 we show the spatial distribution of open clusters together with parameters describing the extinction in $V$ : the total extinction $A_{V}$ and the extinction coefficient $a_{V}=A_{V} / d$.
Since $A_{V}$ characterises the total amount of absorbing matter along the line of sight, the total extinction is increasing with distance. One observes this increase towards to the periphery of Fig. 2 (left panel), and notes its different behaviour in different directions. Generally, the $A_{V}$ distribution can be used for examining the visibility conditions in a given direction and their apparent trends. The extinction coefficient is related to dust and can be used for mapping dust clouds (Fig. 2, right panel).

The total extinction was computed in quadratic areas of $75 \times 75 \mathrm{pc}^{2}$ and then smoothed via a $5 \times 5$ rectangular window i.e., the pattern displayed is averaged over a scale of about $0.4 \mathrm{kpc}$. In contrast to this, for better representation of cloud structures, we smoothed the maximum values of $a_{V}$ (instead of their averages like for $A_{V}$ ) which were determined in each square of $75 \times 75 \mathrm{pc}^{2}$.

In Fig. 2 we show, also, all clusters of our sample with individual values of $A_{V}$ and $a_{V}$ indicated by symbol size (and colour). The same size (colour) scale is used in both panels: the smallest (white) circles mark open clusters with $A_{V}<0.5 \mathrm{mag}$ (left panel) or $a_{V}<0.5 \mathrm{mag} / \mathrm{kpc}$ (right panel); the small (yellow), medium (dark yellow), large (red) and the largest (brown, dotted) circles indicate clusters with $A_{V} / a_{V}$ ranging into $(0.5,1.5],(1.5,2.5],(2.5,3.5]$, and $>3.5 \mathrm{mag}$ or $\mathrm{mag} / \mathrm{kpc}$, respectively.

Some general features of the extinction pattern can be observed in the left panel of Fig. 2. At the location of the Sun we find a cavity with a small extinction of the order of $0.1 \ldots 0.2$, which is transformed into a long transparent $\left(A_{V} \approx 0.2 \ldots 0.3\right)$ corridor located in the directions $l \approx 80^{\circ}$ and $220^{\circ}$ of galactic longitude. Another corridor of about the same transparency $\left(A_{V} \approx 0 \mathrm{~m} 2\right)$ could be seen if we consider only clusters with $Z<-20 \mathrm{pc}$. It is directed towards $l \approx 120^{\circ}$ and $l \approx 300^{\circ}$ from the Sun. 

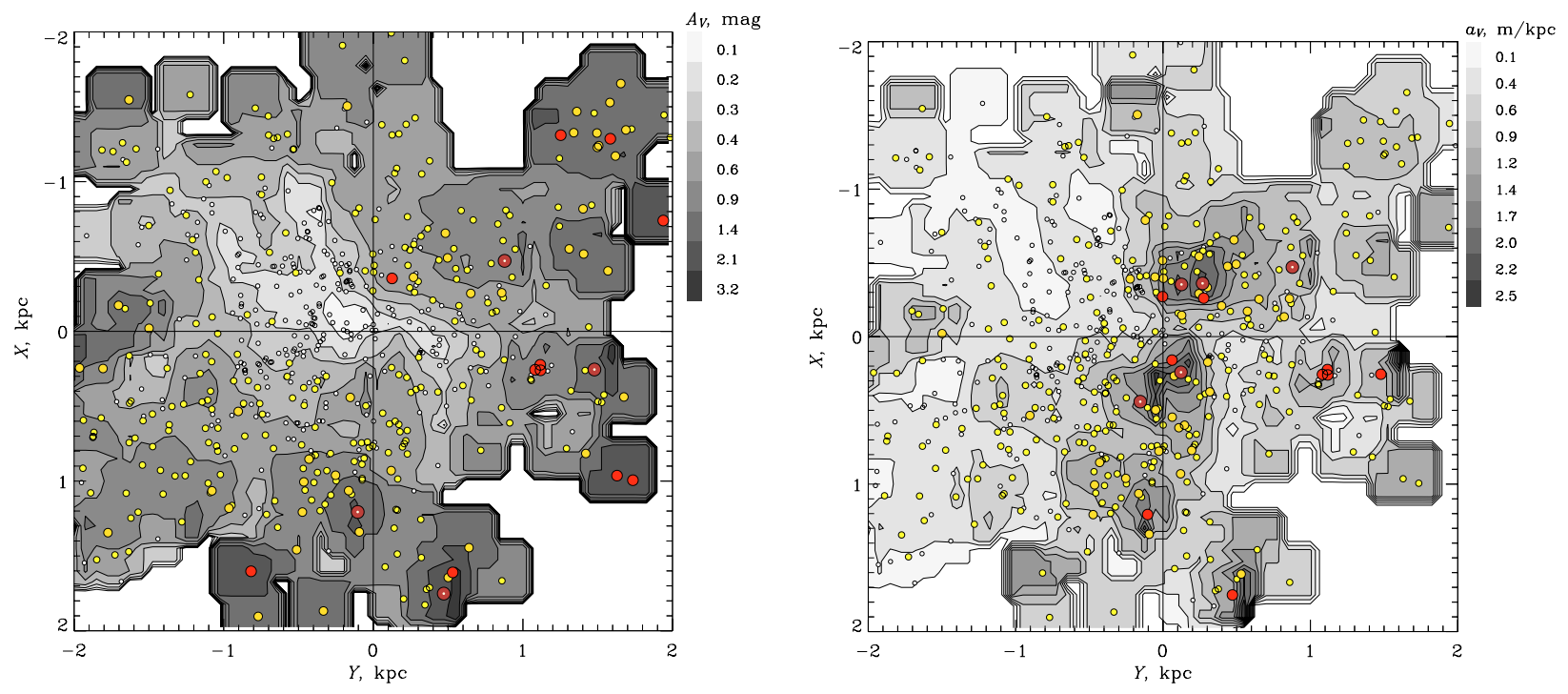

Fig. 2. Open clusters and interstellar extinction. Distributions of the total extinction $A_{V}$ (left panel), and of the extinction coefficient $a_{V}$ (right panel) in the wider Solar neighbourhood. In both panels the contours show $A_{V}, a_{V}$-levels according to the grey-colour scales shown at the right upper corners of the panels. The circles represent open clusters. Their sizes (and colour) correspond to $A_{V}, a_{V}$ of the clusters. The blank areas in the periphery of the plots are due to a lack of clusters in our sample at these locations.

Another feature is the complete absence of clusters (and also extinction data) in some more peripheral areas. Most interesting are the areas at $(X, Y) \approx(-1.2,1) \mathrm{kpc}$ and at $(X, Y) \approx(1.2,1) \mathrm{kpc}$ which manifests a screening effect of massive nearby clouds. Some of these clouds are clearly seen in the right panel, and they are identified with the AurigaTaurus clouds at $X<-0.1 \mathrm{kpc}$, and the Ophiuchus clouds at $X \geq 0 \mathrm{kpc}$. However, the empty area at $(X, Y) \approx(1.2,1) \mathrm{kpc}$ is not caused by the Ophiuchus clouds. Instead, the molecular clouds located in the Aquila Rift region play a more important role here. The clouds are extended farther in the direction of $l \approx 50^{\circ}$ (Dame et al. 2001), and are not seen in our map because the known clusters in this direction are too faint in the optical to be identified in the ASCC-2.5 .

The impact of the clouds on the visible distribution of the clusters is not limited to the Solar vicinity, only. Their shadow can be seen at larger distances and they influence locally the visibility of such structures as the Sagittarius and Perseus arms at some kpc away from the Sun (see Sect. 3.4). Although the spatial density of open clusters is lower than the density of individual stars, the extinction pattern obtained is in a good agreement with most of the previous publications based on data of field stars.

\subsection{Distribution of clusters perpendicular to the Galactic plane}

Distribution along the $Z$-axis is usually described in terms of the barometric formula given in Eq. (1) where $D\left(Z_{0}\right)$ is the density at the position of the symmetry plane $Z_{0}$ and $h_{Z}$ is the scale height:

$D(Z)=D\left(Z_{0}\right) \exp \left\{-\frac{\left|Z-Z_{0}\right|}{h_{Z}}\right\}$
The distribution parameters $Z_{0}, h_{Z}$ and $D\left(Z_{0}\right)$ were computed with data on 259 clusters located within the completeness limit determined in Sect. 3.1. Additionally, we considered the distributions of clusters of the age groups defined in Sect. 3.1. Further, we separated "field" subsamples i.e., excluded probable kinematic members of the cluster complexes discussed in Sect. 5. The corresponding distributions along the $Z$-axis were constructed with a step of $1 \mathrm{pc}$ and then smoothed by use of a rectangular 15-point filter. The results are shown in Fig. 3.

Due to poor statistics (the number of clusters in the different groups varies from 45 to 259) and large density fluctuations, a simultaneous solution of Eq. (1) did not yield a stable and evident result. Therefore, we computed the distribution parameters in two steps. At first, the position of the symmetry plane was assumed to be a simple average in each group. The medians and modes were also considered but the results did not differ significantly. For each sample, the standard deviation $\sigma_{Z}$ of $\left|Z-Z_{0}\right|$ was determined as a statistical measure of cluster scattering. In a second step, the parameters $D\left(Z_{0}\right)$ and $h_{Z}$ were derived by use of the integrated form of Eq. (1):

$\Sigma(Z)=2 D\left(Z_{0}\right) h_{Z}\left(1-\exp \left\{-\frac{\left|Z-Z_{0}\right|}{h_{Z}}\right\}\right)$

where $\Sigma(Z)$ is the surface density of clusters in a layer with width $2\left|Z-Z_{0}\right|$. The parameters were found from a non-linear fit of Eq. (2) to the observed distributions. The results with corresponding rms errors are given in Table 1.

According to Fig. 3 and Table 1, open clusters form an extremely flat system in the Solar neighbourhood. The distribution demonstrates non-regular behaviour with many peaks and gaps. We attribute this to a possible impact of interstellar clouds on the apparent cluster distribution (see also Sect. 3.2). Although varying from one sample to another, the symmetry plane of the cluster system is located below the $(X, Y)$ plane. This finding indicates the position of the Sun above the 
Table 1. Distribution of the clusters along the $Z$-axis within a cylinder of radius of $d_{x y}^{c}$.

\begin{tabular}{|c|c|c|c|c|c|c|c|c|}
\hline \multirow[t]{2}{*}{ Parameter } & \multirow[t]{2}{*}{ all } & \multirow[t]{2}{*}{ all-"field" } & \multicolumn{6}{|c|}{ Cluster age samples } \\
\hline & & & $\mathrm{T} 1$ & T1-“field” & $\mathrm{T} 2$ & T3 & T3-“field" & $\mathrm{T} 4$ \\
\hline$Z_{0}, \mathrm{pc}$ & $-22 \pm 4$ & $-20 \pm 5$ & $-39 \pm 8$ & $-29 \pm 9$ & $-28 \pm 9$ & $-8 \pm 8$ & $-7 \pm 11$ & $-15 \pm 10$ \\
\hline$N$ & 259 & 211 & 72 & 49 & 45 & 76 & 51 & 66 \\
\hline$D\left(Z_{0}\right), \mathrm{kpc}^{-3}$ & $1015 \pm 45$ & $821 \pm 40$ & $263 \pm 21$ & $200 \pm 20$ & $209 \pm 24$ & $376 \pm 34$ & $232 \pm 25$ & $236 \pm 20$ \\
\hline$h_{Z}, \mathrm{pc}$ & $56 \pm 3$ & $56 \pm 3$ & $61 \pm 6$ & $54 \pm 6$ & $48 \pm 6$ & $44 \pm 4$ & $48 \pm 6$ & $61 \pm 6$ \\
\hline$\sigma_{Z}, \mathrm{pc}$ & $74 \pm 3$ & $75 \pm 3$ & $72 \pm 6$ & $68 \pm 7$ & $63 \pm 6$ & $71 \pm 5$ & $78 \pm 8$ & $82 \pm 7$ \\
\hline
\end{tabular}

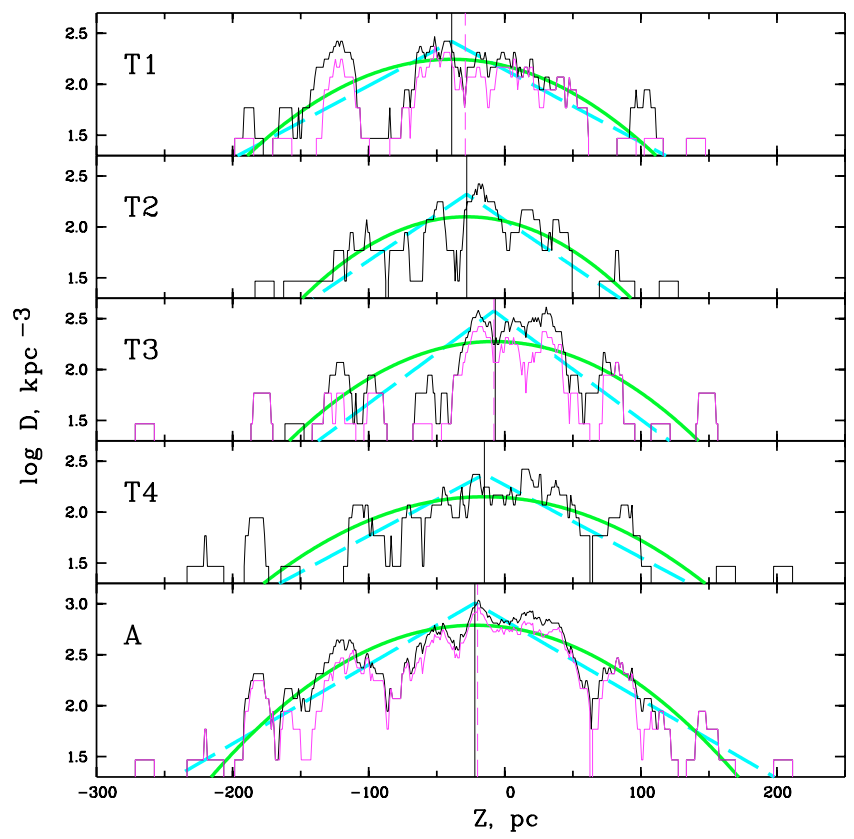

Fig. 3. Distribution of clusters perpendicular to the Galactic plane. The distributions are constructed for clusters within the completeness limit of 850 pc. Panel A: all clusters, panels T1-T4: the 4 age samples (from T1 to T4) defined in Sect. 3.1. The thin black curves indicate smoothed spatial densities. The thin (magenta) curves in panels T1, T3 and A mark "field" subsamples of the corresponding ages. The vertical solid lines give positions $\left(Z_{0}\right)$ of symmetry planes, and the dashed vertical lines show $Z_{0}$ of "field" subsamples. The thick solid curves correspond to Gaussian distributions computed with parameters listed in Table 1. Thick long-dashed lines give the barometric distributions fitted to observations.

physical plane of the system of open clusters. The derived displacement $Z_{0}=22 \pm 4 \mathrm{pc}$ is in good agreement with recent results obtained from studies of other young Population I objects: according to Reed (2005) the position of the symmetry plane of OB stars within $1200 \mathrm{pc}$ from the Sun is located at $Z_{0}=19.5 \pm 2.2 \mathrm{pc}$, whereas the symmetry plane of the dust layer is at $Z_{0}=22.8 \pm 3.3 \mathrm{pc}$ (Joshi 2005). The scale heights vary within 50-60 pc without an evident trend with respect to the ages of clusters (the youngest (T1) and the oldest (T4) groups have the largest $h_{Z}$ value). Although the standard deviations $\sigma_{Z}$ are always larger than the scale heights, the corresponding Gaussian and barometric distributions do not differ significantly (see Fig. 3).
We found the total volume and surface densities of clusters in the plane of symmetry to be $D\left(Z_{0}\right)=1015 \mathrm{kpc}^{-3}$ and $\Sigma=114 \mathrm{kpc}^{-2}$, respectively. The latter number considerably (by a factor of about 5) exceeds the surface density of open clusters which is inferred as $\Sigma=24.9 \mathrm{kpc}^{-2}$ from Janes et al. (1988, Table 8). This discrepancy can be explained by mainly two reasons: a) a significant number of clusters within $0.85 \mathrm{kpc}$ in our sample is missing in the sample of Janes et al. (1988), and b) the completeness of their cluster sample is overestimated by Janes et al. (1988). A combination of these effects should increase their result for the surface density by a factor of 3-6.

Provided that the cluster density distribution in the Galactic disk can be described by a radial density profile with a scale length of the disk $h_{1}$, we estimate the total number of clusters $N_{\text {tot }}$ in the Galaxy as

$N_{\text {tot }}=2 \pi \Sigma \int_{0}^{R_{\mathrm{G}}^{\lim }} \exp \left(-\frac{R_{\mathrm{G}}-R_{G \odot}}{h_{1}}\right) R_{\mathrm{G}} \mathrm{d} R_{\mathrm{G}}$.

Here $R_{\mathrm{G}}$ is the distance from the centre of the Galaxy, $R_{\mathrm{G}}^{\lim }$ is the radius of the Galactic disk, and $h_{1}$ is the scale length, adopted from Bahcall \& Soneira (1980) to be $h_{1}=3.5 \mathrm{kpc}$. With $R_{\mathrm{G}}^{\lim }=15 \mathrm{kpc}$, we derive the total number of open clusters in the Galaxy as $N_{\text {tot }}=93000$. Within a reasonable range of the parameters $R_{\mathrm{G}}^{\lim }=13-20 \mathrm{kpc}$, and $h_{1}>3 \mathrm{kpc}$ the above estimate is valid within $20 \%$. For smaller values of $h_{1}$, the expected number of open clusters would come out to be considerably larger.

\subsection{Distribution of open clusters in the Galactic plane}

The distribution of open clusters in the Galactic plane is presented in Fig. 4. There we show also some 300 clusters from the catalog DLAM (version 2.0) which have distances and ages, but are missing in our sample since they are mostly too faint to be identified in the ASCC-2.5 data. Only about 30 of them are located within the completeness area of our sample. It turned out that all these 30 clusters did not fulfill our criteria for being real open clusters and were thus excluded from the sample. The retained clusters shown in Fig. 4 append the current distribution at larger distances.

Until now the pattern of the spiral structure of the Galaxy is not clearly established. In order to fit the observations, different models have been proposed which suggest two, three or 


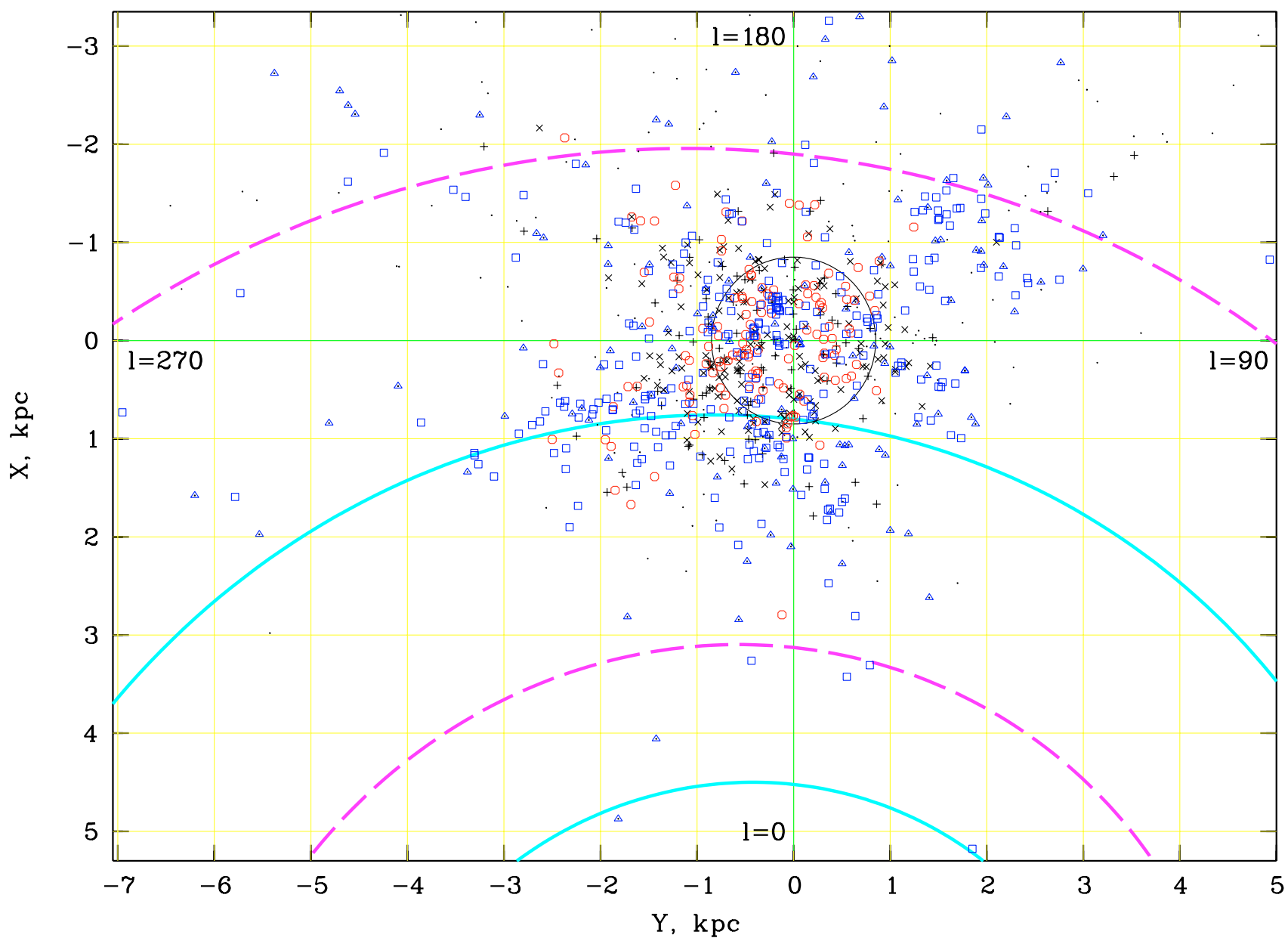

Fig. 4. Distribution of open clusters in the $X Y$-plane centered at the location of the Sun. Blue squares mark T1 clusters, black plusses - T2, red circles - T3, and black crosses - T4 clusters. Dots indicate the positions of clusters with known distances and ages from the DLAM catalogue (v2.0), which are not included in our sample. The youngest $(\log t<7.9)$ of these clusters are marked by triangles. Long dashed and solid thick curves are the grand design spiral arms with a pitch-angle of $-6^{\circ}$ fitted to young clusters in the Perseus and Carina-Sagittarius regions. The large circle around the Sun indicates the completeness area of our sample.

four spiral arms, with pitch angles ranging from $-5^{\circ}$ to $-21^{\circ}$ (see Valée 1995, for reference). Also, many attempts were undertaken to discuss the apparent distribution of open clusters in the Galactic plane with their relation to a local spiral pattern (see e.g. Becker 1963; Janes et al. 1988). Since the clusters of our sample have homogeneous distances and ages, we can try to fit their distribution by a spiral pattern, but we must take into account that the real distribution of open clusters in the optical is hidden by lumpy extinction, and we see distant clusters only by chance through transparent windows (cf. Figs. 2 and 4).

In Fig. 4 we show the grand design pattern of logarithmic spirals with a pitch angle $p=-6^{\circ}$ fitted to the Perseus and Carina-Sagittarius arms. The choice of the pitch-angle is dictated by our intention to provide a reasonable fit of a simple pattern (i.e., a two-arm spiral) to the observed distribution of young clusters at different galactocentric distances. We have succeeded in getting a reasonable agreement along the whole Perseus arm (from $l \approx 90^{\circ}$ to about $270^{\circ}$ ), the CarinaSagittarius arm (from $l \approx 300^{\circ}$ to about $360^{\circ}$ ), and its extension in Vulpecula at about $(X, Y)=(1,1.5) \mathrm{kpc}$. Taking into account relative errors of distances of about $15 \%$, it is not excluded that a few distant clusters at about $X=3 \mathrm{kpc}$ and $X=5 \mathrm{kpc}$ trace the inner convolutions of the Perseus and Carina-Sagittarius spirals.

No open clusters are visible at the expected position of the Carina-Sagittarius arm at $l=20-55^{\circ}$. We explain this by massive molecular clouds located in this direction (see the discussion before). According to Bica et al. (2003b) the gap is well filled by 45 embedded clusters detected in the 2MASS atlas. However, they cannot be used as tracers of the spiral arm since reliable distances are still unknown for them.

\section{Kinematics of open clusters}

The first efforts to transform known proper motions of open clusters to a common reference system was undertaken by van Schewick (1971) who derived absolute proper motions of about 70 open clusters in the FK4 system. Lyngå (1982) provided radial velocities for 108 open clusters, but only for a few tens of them all three vectors of the space velocity could be obtained at that time. Due to the Hipparcos observations and to the development of coravel-type spectrovelocimeters, this situation has improved considerably. Currently, all 652 clusters of our 
sample have accurate proper motions in the Hipparcos system and uniform heliocentric distances. For about $55 \%$ of them, the mean radial velocities $(R V)$ are also available (Paper II). Since the kinematic data of clusters are generally more accurate than these of single field stars, we make use of this advantage to learn about the kinematics of the Galactic disk, in more detail. On the other hand, we should keep in mind that the subsample of open clusters with complete kinematic data is biased towards younger clusters: $R V \mathrm{~s}$ are available for about $75 \%$ of clusters younger than $\log t=8.3$, whereas for older clusters, radial velocities are known for only $31 \%$ of them. This has to be taken into account if one compares the kinematic parameters derived from proper motions (or tangential velocities) of 652 clusters or from the subsample of clusters with proper motions and radial velocities.

In the following, we distinguish three types of space velocities. Firstly, these are observed velocities which are computed directly from COCD proper motions and radial velocities. For each cluster, this space velocity contains systematic components due to Galactic differential rotation and due to Solar motion with respect to the centroid of the open cluster system, as well as a peculiar motion of a cluster with respect to this centroid. The second type are velocities corrected for Galactic differential rotation, and, finally, the third type are velocities corrected both for Galactic differential rotation and for Solar motion (i.e., the peculiar velocities of clusters with respect to the centroid of the open cluster system).

As a first step, we determine kinematic parameters describing the basic motions of the system of open clusters in the Galactic disk: the motion with respect to the Sun, and the differential rotation around the Galactic centre. The Solar motion components $U_{\odot}, V_{\odot}, W_{\odot}$ were derived with the complete kinematic data of 148 clusters located within $d_{x y}^{\mathrm{c}} \leq 0.85 \mathrm{pc}$ as:

$U_{\odot}=-\bar{U}, \quad V_{\odot}=-\bar{V}, \quad W_{\odot}=-\bar{W}$

with $U, V, W$ computed from proper motions and radial velocities with

$$
\begin{aligned}
U & =k d\left(\mu_{1} \sin l+\mu_{b} \cos l \sin b\right)-R V \cos l \cos b \\
V & =k d\left(-\mu_{1} \cos l+\mu_{b} \sin l \sin b\right)-R V \sin l \cos b \\
W & =-k d \mu_{b} \cos b-R V \sin b
\end{aligned}
$$

where $k$ is the scale factor converting angular motions into linear velocities. The Oort's constants $A$ and $B$ of the Galactic rotation were determined from the proper motions $\mu_{1}, \mu_{b}$ of 581 clusters with distances less than $d_{x y} \leq 2500$ pc by a LSQ-solution of the equations:

$$
\begin{aligned}
k d \mu_{1}= & \left(U_{\odot} \sin l-V_{\odot} \cos l\right)+(A \cos 2 l+B) \cos b, \\
k d \mu_{b}= & \left(U_{\odot} \sin b \cos l+V_{\odot} \sin b \sin l-W_{\odot} \cos b\right) \\
& -\frac{A}{2} \sin 2 b \sin 2 l .
\end{aligned}
$$

\begin{tabular}{|c|c|c|}
\hline Parameter & Value & rms error \\
\hline$U_{\odot}, \mathrm{km} \mathrm{s}^{-1}$ & +9.44 & 1.14 \\
\hline$V_{\odot}, \mathrm{km} \mathrm{s}^{-1}$ & +11.90 & 0.72 \\
\hline$W_{\odot}, \mathrm{km} \mathrm{s}^{-1}$ & +7.20 & 0.42 \\
\hline$\sigma_{U}, \mathrm{~km} \mathrm{~s}^{-1}$ & 13.86 & 0.81 \\
\hline$\sigma_{V}, \mathrm{~km} \mathrm{~s}^{-1}$ & 8.75 & 0.51 \\
\hline$\sigma_{W}, \mathrm{~km} \mathrm{~s}^{-1}$ & 5.05 & 0.30 \\
\hline$\sigma_{U}: \sigma_{V}: \sigma_{W}$ & 1:0.63:0.36 & \\
\hline$\overline{R_{\mathrm{a}}}, \mathrm{kpc}$ & 8.631 & 0.034 \\
\hline$\sigma_{R_{\mathrm{a}}}, \mathrm{kpc}$ & 0.427 & 0.024 \\
\hline$\overline{R_{\mathrm{p}}}, \mathrm{kpc}$ & 6.706 & 0.056 \\
\hline$\sigma_{R_{\mathrm{p}}}, \mathrm{kpc}$ & 0.682 & 0.040 \\
\hline$\overline{Z_{\max }}, \mathrm{kpc}$ & 0.260 & 0.016 \\
\hline$\sigma_{Z_{\max }}, \mathrm{kpc}$ & 0.189 & 0.011 \\
\hline $\bar{e}$ & 0.127 & 0.003 \\
\hline$\sigma_{\mathrm{e}}$ & 0.037 & 0.002 \\
\hline$A, \mathrm{~km} \mathrm{~s}^{-1} \mathrm{kpc}^{-1}$ & +14.5 & 0.8 \\
\hline$B, \mathrm{~km} \mathrm{~s}^{-1} \mathrm{kpc}^{-1}$ & -13.0 & 1.1 \\
\hline
\end{tabular}

A rigorous solution of Eq. (5) assumes velocity components corrected for Galactic rotation, whereas the velocities in Eq. (6)
Table 2. Kinematic and orbital parameters of the sub-system of open clusters in the Galaxy.

should be free from the Solar motion. In order to fit these introduced corrections separately, we solved the equation system (4)-(6) by iteration. Only a few iterations were necessary before convergence was achieved.

In the next step, the space velocities of 148 clusters within $d_{x y} \leq 0.85 \mathrm{kpc}$ corrected for Solar motion and Galactic rotation were used for computing the parameters $\left(\sigma_{U}, \sigma_{V}, \sigma_{W}\right)$ of the velocity ellipsoid. Assuming the form of the Galactic potential proposed by Saio \& Yoshii (1979), we also derived elements of their Galactic box orbits. This includes the apocentre and pericentre distances $R_{\mathrm{a}}$ and $R_{\mathrm{p}}$, the eccentricity $e$ and the maximum vertical distance $Z_{\max }$ which a cluster can reach in its orbital motion. The results are given in Table 2 .

Figure 5 shows the distribution of kinematic data of open clusters versus Galactic longitude for different ranges of distances as indicated at the top of the figure. The curves mark systematic contributions of Solar motion and Galactic rotation to the observed velocities of open clusters which are derived with the parameters in Table 2 . Since this systematic velocity component is a function of distances, we show its contribution with the dashed and solid curves for the minimum and maximum distances in a given distance range, respectively. The rms errors for proper motions and radial velocities are taken from the COCD, the errors of tangential velocities are derived as:

$\varepsilon_{\mathrm{T}}^{2}=k^{2} d^{2} \mu^{2}\left(\delta_{\mu}^{2}+\delta_{d}^{2}\right)$,

where $\delta_{\mu}=\varepsilon_{\mu} / \mu, \delta_{d}=\varepsilon_{d} / d$ are the relative errors of a proper motion and distance. The relative error of a proper motion $\delta_{\mu}$ is computed from the individual data of clusters, whereas $\delta_{d}$ is assumed to be $15 \%$ of a distance (see Paper II).

In an ideal case, the observed velocities should be located between the dashed and solid curves in each panel of Fig. 5. 


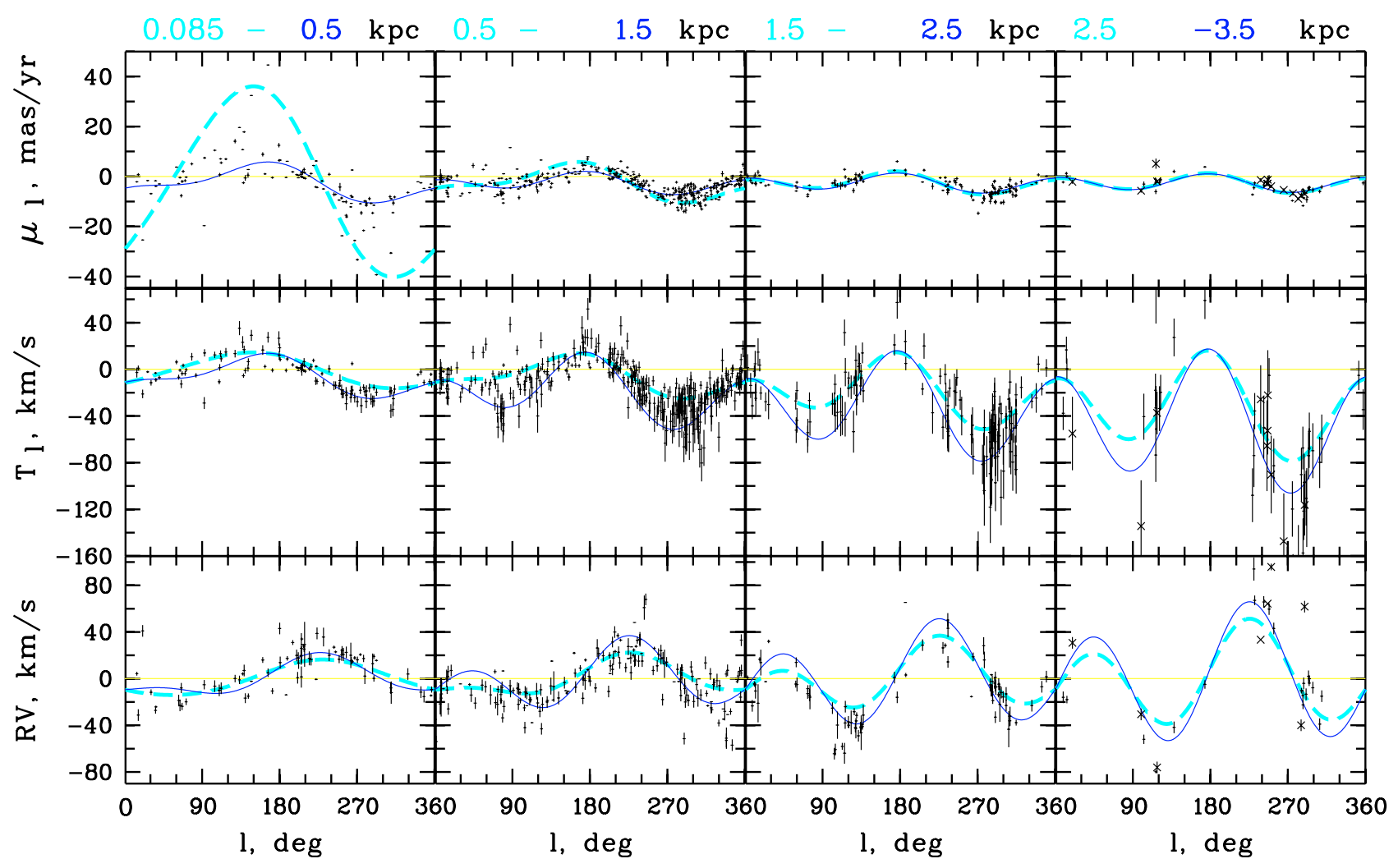

Fig. 5. Observed kinematic data of open clusters versus Galactic longitude for four ranges of distances (as indicated at the top). The upper panels are for proper motions, the middle - for tangential velocities, and the bottom - for radial velocities. The parameters of individual clusters are marked by thin horizontal strokes together with the vertical error bars (in the $R V$ panel the error bars are given only for clusters for which the rms-errors could be estimated). The crosses in the rightmost panels indicate clusters with $d>3.5 \mathrm{kpc}$. For a given distance range, the curves show systematic velocity components due to Solar motion and Galactic rotation computed with the parameters $U_{\odot}, V_{\odot}, W_{\odot}$, A and B in Table 2 . The thick dashed curves correspond to the contributions at the smallest distance of a given distance range, whereas the thin solid curves show this contribution at the largest distance. For example, in the upper left panel the dashed curve shows systematic variations versus longitude in proper motions expected for a cluster at $0.085 \mathrm{kpc}$ from the Sun, whereas the solid curve shows this variations for a cluster at $0.5 \mathrm{kpc}$.

Deviations significantly larger than the individual rms errors indicate peculiarities in cluster motions. For example, a few clusters at longitudes $44 \ldots 124^{\circ}$ and distances $0.5 \ldots 1.5 \mathrm{kpc}$ show unusually large proper motions and tangential velocities. We found that all of them are older than $\log t=8.6$. With the exception of NGC 6991 their radial velocities are unknown, so we cannot determine the complete space velocity vector. Fortunately, their location close to $l=90^{\circ}$ allows us to estimate the $U$-components, and we found that these clusters move towards the Galactic anticentre: $U$-velocities corrected for the Galactic rotation are from -30 to $-50 \mathrm{~km} \mathrm{~s}^{-1}$. Direct calculations of the velocity vector for NGC 6991 give a similar radial component $U=-58 \mathrm{~km} \mathrm{~s}^{-1}$. The age of the clusters as well as their motion towards the Galactic anticentre remind us of the Hyades $\left(\log t=8.90, U=-43.5 \mathrm{~km} \mathrm{~s}^{-1}\right)$. We consider this cluster group in Sect. 5 in more detail.

Oort's constants of Galactic rotation are related to the distance of a cluster from the Galactic centre $R_{\mathrm{G}}$, and to the angular velocity $\Omega$ of the disk as:

$A=-\frac{R_{\mathrm{G}, 0}}{2}\left(\frac{\mathrm{d} \Omega}{\mathrm{d} R_{\mathrm{G}}}\right)_{0}$,

$B=A-\Omega_{0}$.
Assuming the galactocentric distance of the Sun $R_{\mathrm{G}, 0}=$ $8.5 \mathrm{kpc}$, we obtain the angular velocity of the Galactic rotation $\Omega_{0}=A-B=27.5 \pm 1.3 \mathrm{~km} \mathrm{~s}^{-1} \mathrm{kpc}^{-1}$ and the corresponding rotation velocity of the system of open clusters as $233.8 \mathrm{~km} \mathrm{~s}^{-1}$.

This result is in a good agreement with the rotation parameters derived from Hipparcos data over a similar range of ages (less than 1 Gys). For example, Feast \& Whitelock (1997) obtained $(A, B)=(14.82 \pm 0.84,-12.37 \pm 0.64) \mathrm{km} \mathrm{s}^{-1} \mathrm{kpc}^{-1}$ from kinematic data on 220 Galactic cepheids located mainly within $4 \mathrm{kpc}$ from the Sun. Using data on about 2000 O-B stars, Torra et al. (2000) found $(A, B)=(11.8 \pm 0.4,-12.3 \pm$ $0.4) \mathrm{km} \mathrm{s}^{-1} \mathrm{kpc}^{-1}$ for $d \leq 2 \mathrm{kpc}$. From data on about 1820 O-B5 stars within $1 \mathrm{kpc}$ from the Sun, Branham (2002) derived $(A, B)=(+14.9 \pm 0.8,-15.5 \pm 0.7) \mathrm{km} \mathrm{s}^{-1} \mathrm{kpc}^{-1}$.

Also, the parameters of Solar motion derived with open clusters agree quite well with previous results for the moderately young disk. Based on a kinematically unbiased sample from the Hipparcos catalogue in the Solar velocity, Dehnen \& Binney (1998) determined the Solar motion with respect to the $\operatorname{LSR}$ as $(U, V, W)_{\odot}=(10.00 \pm 0.36,5.25 \pm$ $0.62,7.17 \pm 0.38) \mathrm{km} \mathrm{s}^{-1}$. Whereas the $U$ and $W$-components are in a perfect agreement with the results derived with open clusters, the rotation component is about two times 
smaller than in Table 2. The difference is mainly caused by the different evolution parameters of the samples used. The sample of Dehnen \& Binney contains Main Sequence stars from the Solar neighbourhood with $(B-V) \lesssim 1.2$ i.e., stars of different ages, from a few million years up to 10 Gyr. As we already mentioned before, our cluster sample represents the population of the moderately young disk. (see also Sect. 6). Indeed, comparing our results with that of Torra et al. (2000) who found $(U, V, W)_{\odot}=(11.00 \pm 0.2,12.9 \pm 0.2,6.8 \pm 0.1) \mathrm{km} \mathrm{s}^{-1}$ with OB stars, we see a good agreement for all components of the Solar motion. We conclude that both systems of young stars and of open clusters move similarly.

Since the age for each open cluster of our sample was determined by the same method, we can check whether real trends in kinematic parameters exist, depending on ages. The most interesting trend is a dependence of the cosmic (or true) velocity dispersion on the cluster age. Due to the inhomogeneity of the cluster $R V \mathrm{~s}$, it is rather difficult to estimate a realistic contribution of the rms errors in the measurements to the observed dispersion of space velocities. Therefore, we prefer to use the dispersion of tangential velocities which can be properly corrected for the rms error of observations. Let $\varepsilon_{\mathrm{T}}$ be the mean error of tangential velocities of a given cluster sample, then

$\tilde{\sigma}_{\mathrm{T}}^{2}=\sigma_{\mathrm{T}}^{2}-\varepsilon_{\mathrm{T}}^{2}$

where $\tilde{\sigma}_{\mathrm{T}}$ is the wanted cosmic dispersion, and $\sigma_{\mathrm{T}}$ is the dispersion of observed tangential velocities corrected for the Solar motion and Galactic rotation. We consider all clusters within $d_{x y}^{\mathrm{c}}$, and with $\log t$ from 6.7 to 9.5 binned in 10 overlapping groups of ages. The resulting relation is well approximated by the following equations:

$\tilde{\sigma}_{T_{l}}=(1.44 \pm 0.45) \log t_{6}+(3.93 \pm 0.84)$,

$\tilde{\sigma}_{T_{b}}=(1.33 \pm 0.17) \log t_{6}+(1.65 \pm 0.33)$

where $t_{6}$ is the cluster age in units of Myr. For a given $\log t$, $\left(\tilde{\sigma}_{T_{l}}, \tilde{\sigma}_{T_{b}}\right.$ ) represent the expected dispersions of tangential velocities of clusters of this age and describe the ellipse of the velocity dispersion, or simply the velocity ellipse. Since the $T_{l}$ components might be biased by residual rotations, we use the second equation of (10) for estimating the ellipsoid of the cosmic dispersion. Moreover, for a flat system of open clusters the $T_{b}$ component does not differ significantly from $W$. Using the ratio $\left(\sigma_{U}: \sigma_{V}: \sigma_{W}\right)$ in Table 2 , we derive the ellipsoid of the cosmic dispersion as $(7.2: 4.1: 2.6) \mathrm{km} \mathrm{s}^{-1}$ for young open clusters with $\log t=6.7$, and $(17.5: 10.0: 6.3) \mathrm{km} \mathrm{s}^{-1}$ for old clusters with $\log t=9.5$.

The derived relations were compared with recent findings. Dehnen \& Binney (1998) determined dispersions for MS stars in different colour ranges. Their youngest group was selected in the $(B-V)$-range from -0.238 to 0.139 . According to our estimates, the average $\log t$ of these stars should be somewhere between 8.2 and 8.8. Then, from Eq. (10) we derive $\tilde{\sigma}_{W}=4.6 \ldots 5.4 \mathrm{~km} \mathrm{~s}^{-1}$. The corresponding value of Dehnen \& Binney (1998) is $5.46 \pm 0.61 \mathrm{~km} \mathrm{~s}^{-1}$ and fits well to our estimates. According to Torra et al. (2000), the velocity dispersion of $\mathrm{OB}$ stars increases from $4.3 \mathrm{~km} \mathrm{~s}^{-1}$ for about $15 \mathrm{Myr}$ old stars to $5.5 \mathrm{~km} \mathrm{~s}^{-1}$ for stars older than $120 \mathrm{Myr}$. These results are comparable to ours, although they are larger by about $20 \%$, than those derived from Eq. (10).

\section{The local density inhomogeneities}

In this section we discuss the density enhancement of open clusters found in the spatial distribution in the Solar neighbourhood illustrated in Fig. 1. The density peaks are detected neither only in one age group (this would be the case if a peak is a signature of one physical group of clusters), nor in all of them (which would indicate a general property of the cluster distribution). On the contrary, the clusters from groups T2 and $\mathrm{T} 4$ show distributions which are compatible with a constant density of clusters, whereas the samples $\mathrm{T} 1$ and T3 have significant maxima within the completeness area. Moreover, the samples $\mathrm{T} 1$ and $\mathrm{T} 3$ display differences in their density distribution. In addition to the prominent peak at about $0.4 \mathrm{kpc}$, the sample $\mathrm{T} 1$ shows a secondary density excess of lesser significance at lower distances which can be interpreted as evidence for a prolate structure. The sample T3 demonstrates only one, but a broader density excess. In the following we consider the properties of each sample separately, and try to answer the question: are these density peaks manifestations of independent "clusterings" of open clusters? If so, to which degree are they separated from each other, and from the general cluster substratum?

Hereafter, we call such cluster groups "open cluster complexes" (OCCs), in analogy to large star forming complexes which include associations and young clusters (see e.g., Janes et al. 1988; Efremov 1995). We would like to stress that unlike star forming complexes, which are considered as aggregates of objects related to star formation, the OCCs have been primarily identified within a sample of classical open clusters. Of course, some of them could (but need not) coincide with star forming regions. In the following, we refer to clusters, which are not found to be members of OCCs, as "field" clusters of the Galactic disk.

\subsection{Tangential velocity and membership in complexes}

In order to get additional pros and/or contras for the physical nature of OCCs, we consider the distribution of kinematic parameters of open clusters. Since radial velocities have not been measured for all clusters, we restrict ourselves to the study of two-dimensional kinematics only. As the clusters are located at different distances from the Sun, we use tangential velocities instead of proper motions.

In Fig. 6 we compare the distributions of clusters of different ages in the $\left(T_{b}, T_{l}\right)$ diagrams (i.e., the vector point diagram of tangential velocities, the VPD). The $T_{b}, T_{l}$ velocities are corrected for Solar motion and differential rotation of the Galaxy. Here we consider only clusters located within the completeness area $\left(d_{x y} \leq 0.85 \mathrm{kpc}\right)$ and compare their distribution with distribution of clusters in the enhancement areas (in the samples T1 and T3).

A proper selection of kinematic members requires objective criteria taking into account peculiarities of the kinematic data used. Although we may apply a technique of probability determination normally used for the selection of members 


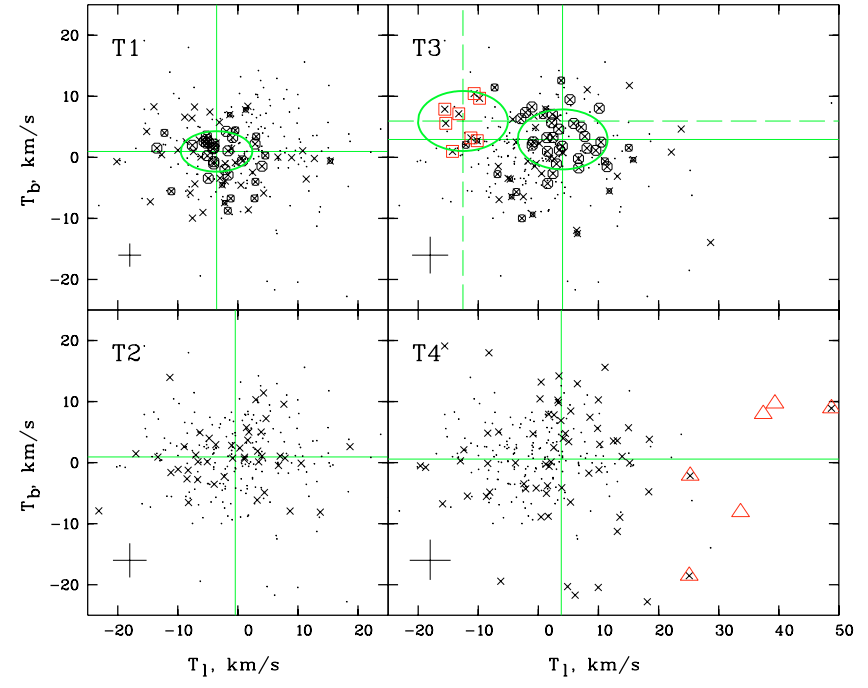

Fig. 6. Vector point diagrams of tangential velocities of open clusters in four age samples (from T1 to T4). The velocities are corrected for Solar motion and differential rotation of the Galaxy. Dots are clusters (independent of age) within the completeness area. The crosses mark clusters of a given age sample. The circles and squares are clusters located within the areas of the density excesses (samples T1 and T3). Their sizes indicate kinematic membership probability to belong to an OCC correspondingly, the largest symbols indicate $1 \sigma$ members, the intermediate circles are $2 \sigma$ members, and the smallest circles are $3 \sigma$ members. The squares (in T3) mark the members of the Perseus-Auriga group (see Sect. 5.3). The triangles (in T4) indicate possible members of the Hyades cluster group (see Sects. 4 and 5.4). The plusses in the bottom-left corners in each panel give the mean errors of tangential velocities of open clusters. The (green) cross-hair indicates the average tangential motion of each group, whereas the ellipses show the velocity ellipses of the complexes.

of a cluster, we must introduce some principal modifications. Determining the members from the proper motion distribution within a small cluster area, we may assume that proper motions of cluster members differ from the average only by their rms errors, and generally, the internal motion is negligibly small compared to uncertainties of proper motions. This assumption is not always true if we consider the common motion of clusters belonging to an OCC. According to Eq. (10), we would expect a cosmic dispersion comparable to the rms errors of the tangential velocities. Due to the definition of the tangential velocities, even clusters with similar space velocities should show different tangential velocities as a function of their galactic coordinates. This effect will introduce an additional spread of data points in the VPD. Since the clusters of our sample represent a flat system, this spread should be more significant in $T_{l}$ than in $T_{b}$. Let us assume an ideal (i.e., without internal rotation and velocity dispersion) stream of clusters located in the Galactic plane and well distributed around the Sun. Then, the dependence of $T_{l}$ on galactic longitude $l$ for each cluster has a sine function:

$T_{l}=-A_{T_{l}} \sin \left(l-l_{0}\right)$

where $l_{0}$ is the apex direction of the stream and $A_{T_{l}}$ is the maximum contribution of the velocity component $T_{l}$ to the space velocity of the stream. An additional rotation will simply make the mean tangential velocity $\bar{T}_{1}$ of a cluster group different from zero. It is self-evident that the spread of tangential velocities in the VPD will be smaller if OCC members are located at similar galactic longitudes as well as when they have low common velocities with respect to the centroid of open clusters. However, in reality the dependence will be more or less disturbed by a spread in the location of clusters along the $Z$-axis, by possible internal motions, uncertainties of kinematic data, and so on. Below we discuss the kinematic behaviour of the complexes in further detail.

Due to the reasons described above, we cannot consider the differences of cluster motions with respect to their group average to be a measure of the membership probability as we did in the case of stars in a cluster. In the case of OCCs, we must consider residual motions of individual clusters with respect to the velocity ellipse defined by a realistic dispersion of tangential velocities.

The membership probability $P_{\mathrm{t}}$ of a cluster to belong to a OCC is computed as

$P_{\mathrm{t}}=\exp \left\{-\frac{1}{2}\left[\left(\frac{\delta T_{1}^{i}}{\varepsilon_{T_{l}}^{i}}\right)^{2}+\left(\frac{\delta T_{b}^{i}}{\varepsilon_{T_{b}}^{i}}\right)^{2}\right]\right\}$

Here $\varepsilon_{\mathrm{T}}^{i}$ are rms errors of tangential velocities of the $i$ th cluster, and $\delta T^{i}$ are residual motions of the cluster with respect to the velocity ellipse of the OCC considered. Depending on the residual motions $\delta T^{i}$, we classify the membership probability as high $\left(\delta T^{i} / \varepsilon_{\mathrm{T}}^{i}<1, P_{\mathrm{t}}>61 \%\right)$, moderate $(1 \leq$ $\left.\delta T^{i} / \varepsilon_{\mathrm{T}}^{i}<2, P_{\mathrm{t}}=14 \%-61 \%\right)$, and low $\left(2 \leq \delta T^{i} / \varepsilon_{\mathrm{T}}^{i}<\right.$ $\left.3, P_{\mathrm{t}}=1 \%-14 \%\right)$. Traditionally, we call such objects $1 \sigma, 2 \sigma$, and $3 \sigma$ members (see Paper I), respectively. Note that all clusters within the velocity ellipse have the highest membership probability $\left(P_{\mathrm{t}}=100 \%\right)$.

The parameters of velocity ellipses were chosen in accordance with Eqs. (10) derived in Sect. 4. The centres of the distribution and membership probabilities were computed in an iterative procedure. As a first approximation of the centres, we used a simple average of the tangential velocities of all clusters at a given age and we derived membership probabilities by Eq. (12). After that, we selected the $1 \sigma$-members to derive the next iteration of the distribution centre. The iterations were stopped when the list of the $1 \sigma$-members did not change in subsequent iterations. The results are shown in Fig. 6 and Table 7.

\subsection{OCC 1: a complex of clusters associated with Gould's Belt}

For the sample T1, we found 37 clusters within $d_{x y}=0.5 \mathrm{kpc}$, with 28 of them located within the main maximum of the density distribution at $d_{x y}=0.325 \ldots 0.5 \mathrm{kpc}$. Recomputing the surface density distribution in terms of numbers of clusters, we infer that $16 \pm 4$ clusters should belong to the field, whereas $20 \pm 4$ clusters are responsible for the density excess. Thus, we may expect about 20 members in a potential OCC. About the same number of clusters is also obtained from the statistics of kinematic membership. Table 7 shows that within $0.5 \mathrm{kpc}$ from the Sun, we found $N=23$ clusters which are $1 \sigma$ members from the distribution of their tangential velocities. We conclude that 


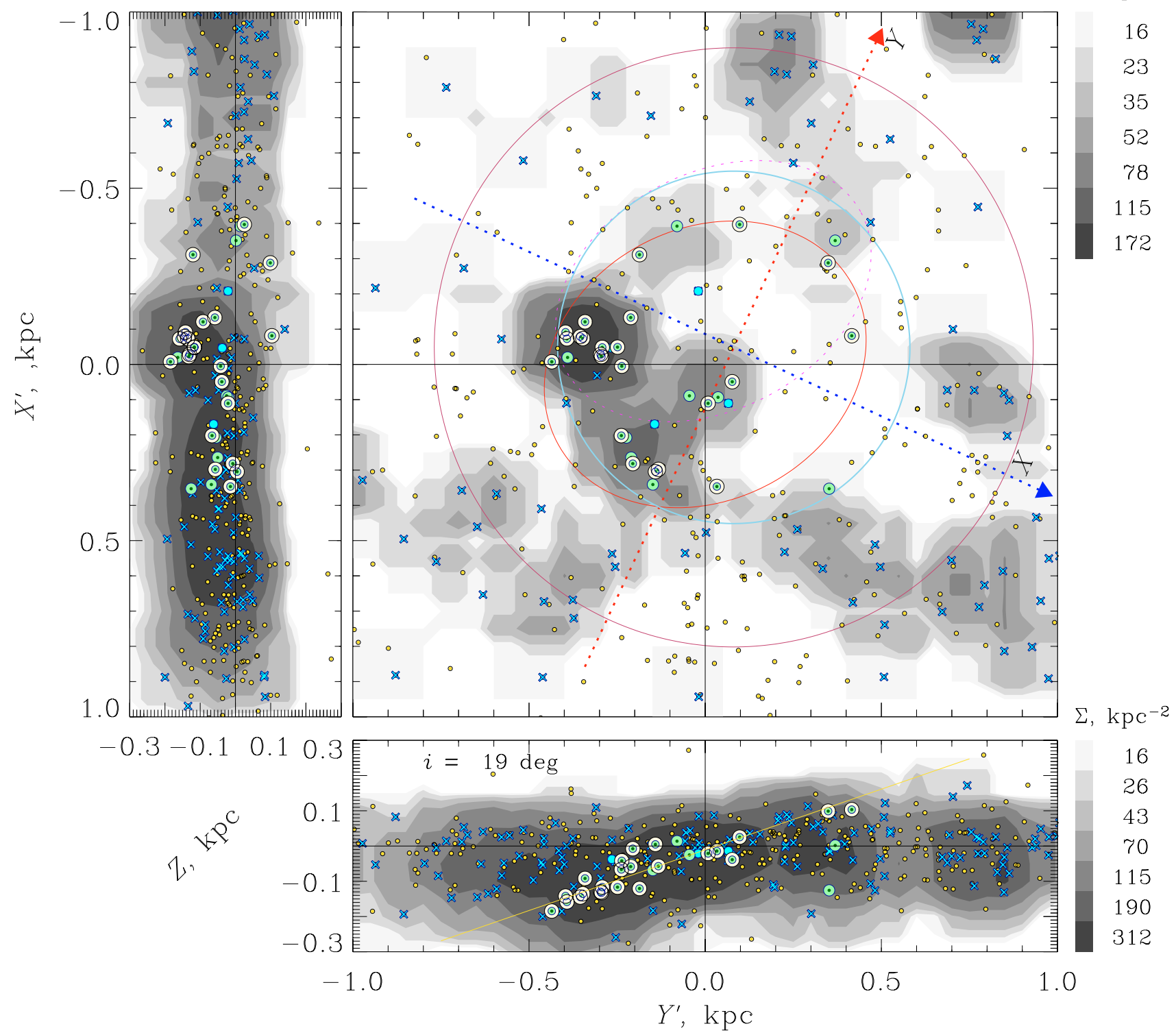

Fig. 7. Spatial distribution of the OCC 1 clusters in 3D-space. The proper coordinate system $X^{\prime}, Y^{\prime}, Z$ of the OCC 1 is described in the text. The coordinate axes $X$ and $Y$ of the Galactic rectangular system are shown with dotted lines. Two circles centered at the origin of the Galactic coordinate system indicate the outer boundary of the area of the enhanced density at $0.5 \mathrm{kpc}$ and the completeness area at $0.85 \mathrm{kpc}$. The solid ellipse outlines the OCC 1 members selected as described in the text. The dashed ellipse represents the Olano (1982) model of gas in Gould's Belt, given here for comparison. The small dots are all the clusters from our sample, the crosses mark T1 clusters, and the filled circles are for member candidates of the OCC 1 . The small (cyan) circles are $3 \sigma$ members, the dotted circles (green) of medium size show $2 \sigma$ members, and the largest dotted circles (light yellow) with a (green) core indicate $1 \sigma$ members. The grey colour contours give the projected density distribution of T1 clusters, derived separately in each panel. The density distribution was computed in windows of $50 \times 50 \mathrm{pc}^{2}$ and smoothed with a 5-point rectangular filter. The rules to the right indicate the corresponding density scales where the $\left(X^{\prime}, Z\right)$ and $\left(Z, Y^{\prime}\right)$ planes share the same grey colour scale. The straight line in the panel $\left(Z, Y^{\prime}\right)$ is the regression line computed with $1 \sigma$ clusters. The resulting inclination $i$ is given in the left upper corner of $\left(Z, Y^{\prime}\right)$ plane.

the $1 \sigma$ threshold for kinematic probability $\left(P_{\mathrm{t}}=61 \%\right)$ separates well the complex members from the field.

The distribution of the OCC 1 candidates in 3D-space is shown in Fig. 7. For convenience, we present the results in a coordinate system related to this cluster group. The coordinate system $X^{\prime}, Y^{\prime}, Z$ is rotated in the Galactic plane to such that the $X^{\prime}$-axis corresponds to the line of nodes of the OCC 1 plane.
The centre of the system is shifted to the apparent centre of the group by $\Delta X=-0.078 \mathrm{kpc}$ and $\Delta Y=-0.053 \mathrm{kpc}$.

According to Fig. 7, the OCC 1 clusters constitute an elongated configuration immersing below the galactic plane in the region of the Orion association by about $200 \mathrm{pc}$, and then rising above the galactic plane by about $100 \mathrm{pc}$ at the opposite direction in the IC 4665 region. The apparent centre of the 
complex is located close to the Sco-Cen group with the clusters Mamajek 1 and Platais 8. On average, the OCC 1 resides below the Galactic plane $(\bar{Z}=-46$ pc for the $1 \sigma$ members $)$. The ascending node of the OCC 1 plane is located near the cluster Alessi 5 at $(l, b)=\left(288^{\circ} .08,-2^{\circ} .05\right)$, whereas the descending node is somewhere near ASCC 127 at $(l, b)=\left(112.26,4^{\circ} .13\right)$. This finding is well compatible with the results by Torra et al. (2000) who derived the longitude of the ascending node of Gould's Belt as $l=275 \ldots 295^{\circ}$ from the Hipparcos data of OB field stars. It seems that the density excess of T1 clusters can be associated with the Gould's Belt complex which is usually believed to contain early-type field stars, associations, or interstellar gas (e.g., see a review by Pöppel 1997).

The OCC 1 has a flat structure, but is not strictly planar. In the $\left(Z, Y^{\prime}\right)$-plane it shows a significant inclination (as the regression solution shows) of $19^{\circ}$ with respect to the Galactic plane. Torra et al. (2000) found an inclination of $i=16 \ldots 22^{\circ}$ from the data on field stars. In the $\left(Z, X^{\prime}\right)$-plane, the complex presents a less pronounced structure. It is a rather smooth transition from the lowest location near NGC 1980 to Stephenson 1 via Vela OB1 and Mamajek 1, and then a steeper rising from IC 348 to IC 4665 via ASCC 127.

In Table 3 we list all $\mathrm{T} 1$ clusters located within $d_{x y}=$ $0.5 \mathrm{kpc}$ from the Sun. Due to its high membership probability $\left(P_{\mathrm{t}}=100 \%\right)$, we extended this list by one Orion cluster (NGC 1980) residing at $d_{x y}=518$ pc. For each cluster in Table 3, we provide the COCD number, the cluster designation, the $(X, Y, Z)$ coordinates, the cluster age, and the kinematic membership probability $P_{\mathrm{t}}$ for belonging to the Gould's Belt complex.

As described above, clusters located within the velocity ellipse got the highest membership probability $P_{\mathrm{t}}=100 \%$. On the other hand, some clusters like e.g. Melotte 20 ( $\alpha$ Per) which were believed to be members of the Gould's Belt system (e.g., see Olano 1982) have a low kinematic probability.

Fortunately, all the $1 \sigma$ members of OCC 1 have full space velocity vectors. Therefore, we can analyse the spatial motion of this complex in more detail. On average, OCC 1 rotates around the Galactic centre at about the same velocity as the centroid of the open cluster system (the relative velocity is $\bar{V}=-0.2 \pm 1.1 \mathrm{~km} \mathrm{~s}^{-1}$ ). The average tangential velocity of the most probable members of the OCC 1 is $\bar{T}_{1}=-3.6 \pm 1.4 \mathrm{~km} \mathrm{~s}^{-1}$ i.e., it differs significantly from zero. Therefore, we may conclude an internal rotation of the OCC 1 complex around its centre near IC 2391 and Mamajek 1, and in the direction of Galactic rotation. Excluding these two clusters which do not contribute to the determination of the internal rotation, we derive $\bar{T}_{1}=-2.9 \pm 0.7 \mathrm{~km} \mathrm{~s}^{-1}$. At an average distance of $0.39 \mathrm{kpc}$ from the OCC 1 centre, one obtains the corresponding angular velocity as $\omega=-8.6 \pm 2.2 \mathrm{~km} \mathrm{~s}^{-1} \mathrm{kpc}^{-1}$. This is considerably lower (in absolute units) than the value $\omega \approx-20 \mathrm{~km} \mathrm{~s}^{-1} \mathrm{kpc}^{-1}$ derived by Bobylev (2004) from the kinematics of Hipparcos stars proposed to be members of associations and open clusters.

The expansion velocity of the OCC 1 computed as a residual motion along a radius-vector projected onto the $X Y$ plane and with origin in the OCC 1 centre is $V_{r}=2.1 \pm 1.1 \mathrm{~km} \mathrm{~s}^{-1}$. This seems to be insignificant. The latter conclusion confirms
Table 3. Candidates of the OCC 1 (the Gould's Belt complex).

\begin{tabular}{|c|c|c|c|c|c|c|}
\hline COCD & Name & $\begin{array}{r}X \\
\mathrm{kpc}\end{array}$ & $\begin{array}{r}Y \\
\mathrm{kpc}\end{array}$ & $\begin{array}{r}Z \\
\mathrm{kpc}\end{array}$ & $\begin{array}{r}\log t \\
\mathrm{yr}\end{array}$ & $\begin{array}{l}P_{\mathrm{t}} \\
\%\end{array}$ \\
\hline 41 & Stock 23 & -0.291 & 0.244 & 0.014 & 7.51 & 25 \\
\hline 42 & Melotte 20 & -0.159 & 0.102 & -0.021 & 7.55 & 2 \\
\hline 46 & IC 348 & -0.354 & 0.125 & -0.120 & 7.79 & 84 \\
\hline 68 & Collinder 65 & -0.301 & -0.047 & -0.058 & 7.41 & 100 \\
\hline 72 & Collinder 69 & -0.413 & -0.113 & -0.092 & 6.76 & 100 \\
\hline 73 & NGC 1981 & -0.334 & -0.178 & -0.130 & 7.50 & 100 \\
\hline 74 & NGC 1976 & -0.329 & -0.183 & -0.132 & 7.71 & 100 \\
\hline 75 & NGC 1977 & -0.415 & -0.225 & -0.164 & 7.08 & 40 \\
\hline 76 & NGC 1980 & -0.451 & -0.255 & -0.184 & 6.67 & 100 \\
\hline 77 & Collinder 70 & -0.338 & -0.158 & -0.117 & 6.71 & 100 \\
\hline 80 & Sigma Ori & -0.340 & -0.172 & -0.119 & 6.82 & 5 \\
\hline 91 & Platais 6 & -0.313 & -0.148 & -0.038 & 7.79 & 1 \\
\hline 95 & NGC 2232 & -0.266 & -0.183 & -0.042 & 7.49 & 100 \\
\hline 126 & Collinder 132 & -0.183 & -0.362 & -0.066 & 7.51 & 100 \\
\hline 133 & Collinder 135 & -0.112 & -0.292 & -0.062 & 7.54 & 11 \\
\hline 136 & Collinder 140 & -0.168 & -0.361 & -0.055 & 7.57 & 15 \\
\hline 159 & NGC 2451A & -0.056 & -0.178 & -0.025 & 7.76 & 36 \\
\hline 162 & NGC 2451B & -0.132 & -0.406 & -0.050 & 7.88 & 19 \\
\hline 182 & Vel OB2 & -0.048 & -0.404 & -0.057 & 7.26 & 100 \\
\hline 183 & NGC 2547 & -0.044 & -0.450 & -0.068 & 7.70 & 23 \\
\hline 190 & vdB-Hagen 23 & -0.120 & -0.420 & -0.008 & 7.14 & 100 \\
\hline 202 & IC 2391 & 0.001 & -0.175 & -0.021 & 7.88 & 73 \\
\hline 204 & Mamajek 1 & 0.037 & -0.090 & -0.039 & 6.90 & 100 \\
\hline 210 & Trumpler 10 & -0.052 & -0.414 & 0.005 & 7.38 & 100 \\
\hline 216 & Platais 8 & 0.020 & -0.147 & -0.020 & 7.75 & 27 \\
\hline 259 & IC 2602 & 0.053 & -0.150 & -0.014 & 7.83 & 13 \\
\hline 261 & Alessi 5 & 0.123 & -0.378 & -0.014 & 7.71 & 100 \\
\hline 412 & IC 4665 & 0.290 & 0.171 & 0.103 & 7.63 & 100 \\
\hline 456 & Stephenson 1 & 0.142 & 0.331 & 0.099 & 7.69 & 100 \\
\hline 479 & Roslund 5 & 0.133 & 0.396 & 0.002 & 7.77 & 28 \\
\hline 1016 & ASCC 16 & -0.408 & -0.156 & -0.145 & 6.93 & 100 \\
\hline 1018 & ASCC 18 & -0.439 & -0.178 & -0.159 & 7.12 & 100 \\
\hline 1019 & ASCC 19 & -0.299 & -0.139 & -0.117 & 7.64 & 100 \\
\hline 1020 & ASCC 20 & -0.399 & -0.158 & -0.135 & 7.35 & 100 \\
\hline 1021 & ASCC 21 & -0.451 & -0.163 & -0.142 & 7.11 & 100 \\
\hline 1024 & ASCC 24 & -0.318 & -0.236 & -0.057 & 6.96 & 0 \\
\hline 1089 & ASCC 89 & 0.415 & -0.249 & -0.126 & 7.03 & 42 \\
\hline 1127 & ASCC 127 & -0.132 & 0.323 & 0.025 & 7.82 & 68 \\
\hline
\end{tabular}

the results of Torra et al. (2000) who found an expansion velocity of $1.5 \pm 1 \mathrm{~km} \mathrm{~s}^{-1}$ for young field stars at $d>0.25 \mathrm{kpc}$.

The total velocity dispersion $\sigma_{U V W}$ for the 23 members of the OCC 1

$\sigma_{U V W}^{2}=\left(\sigma_{U}^{2}+\sigma_{V}^{2}+\sigma_{W}^{2}\right) / 3$ 
$\sum, \mathrm{kpc}^{-2}$

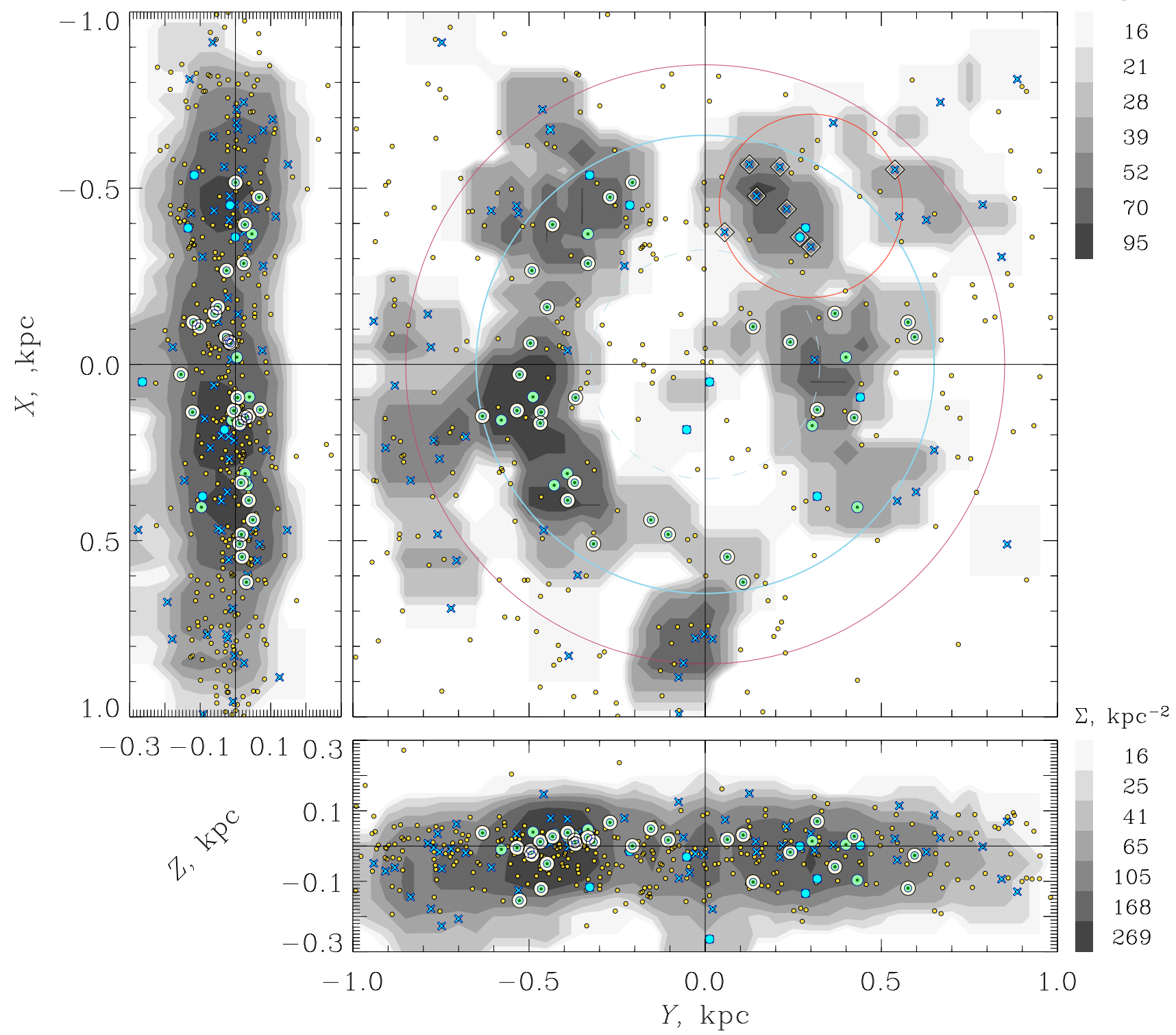

Fig. 8. Spatial distribution of T3 clusters in 3D-space. The coordinates are given in the Galactic cartesian system. The larger circles indicate possible members of OCC2, the diamonds mark members of the Perseus-Auriga group (in order to avoid an overcrowding in the $X, Z$ and $Y, Z$ panels, they are shown together with the corresponding boundary circle only in the $X, Y$ panel). The dashed circle gives the inner edge of the enhancement area. The other designations are the same as in Fig. 7.

is found to be $4.8 \pm 0.7 \mathrm{~km} \mathrm{~s}^{-1}$. This is significantly less than the total dispersion of $7.6 \pm 0.7 \mathrm{~km} \mathrm{~s}^{-1}$ computed from all $69 \mathrm{~T} 1$ clusters in the completeness area. This difference gives an additional confirmation for the distinction of the complex from the general field.

\subsection{OCC 2 and the Perseus-Auriga group: two in one}

According to the cluster counts within $d_{x y}=0.65 \mathrm{kpc}$, the sample T3 contains 53 clusters, with 48 of them in the location of the main density maximum (i.e., at $d_{x y}=0.325 \ldots 0.65 \mathrm{kpc}$ ). The lower density of T3 clusters at $d_{x y}<0.325 \mathrm{kpc}$ from the Sun can well be seen in Figs. 1 and 8. Recomputing the surface density distribution in terms of numbers of clusters, we infer that $33 \pm 6$ of them belong to field clusters and $20 \pm 6$ form the density excess. The number of kinematic candidates of OCC 2 (i.e., $1 \sigma$ members) is 27 (see Table 7). Although a difference of 7 clusters is still within the statistical uncertainty, the disagreement between both estimates is larger than in the OCC 1 case. The average density used for computing the number of field clusters is determined from the distribution of T3 clusters up to $d_{x y}=0.85 \mathrm{kpc}$, and it is slightly higher than the density in the inner part of $d_{x y}<0.325 \mathrm{kpc}$ from the Sun (Fig. 1). Assuming the density in the Solar neighbourhood as being representative for the T3 group, we obtain 20 field clusters and 33 OCC members forming the enhancement, and the excess would be extended somewhat outward of $0.65 \mathrm{kpc}$. Below, we show that, indeed, we found another kinematic group, the Perseus-Auriga group (see Fig. 8 
and Table 7), among the T3 clusters. The complex is very compact in space as well as in the VPD and consists of 8 clusters including one cluster at $d_{x y}=0.77 \mathrm{kpc}$ i.e., outside the main density excess. Consequently, the final census of the T3 sample within $d_{x y}=0.65 \mathrm{kpc}$ is 27 OCC 2 members, 7 members of the Perseus-Auriga group and 19 field clusters. This result coincides well with the number of 20 field clusters estimated before. Although OCC 2 and the Perseus-Auriga group are of the same ages, they show different kinematics and spatial distribution. Therefore we consider these two groups as separate entities.

The average age of $1 \sigma$ members of OCC 2 is $\log t=8.48$, with a standard deviation $\sigma_{\log t}=0.09$. The distribution of the T3 clusters in 3D-space is shown in Fig. 8 in the Galactic cartesian coordinate system $X Y Z$. Kinematic members of OCC 2 form a ring-like structure with an outer diameter of about $1.3 \mathrm{kpc}$, and there are only a few T3 clusters in its inner part. The ring is ripped along the main Galactic meridian, or more exactly along a line at $l \approx 15^{\circ}, l \approx 195^{\circ}$, where no $1 \sigma$ members are found. It even seems that $1 \sigma$ members form two subgroups which are separated by this line. For convenience, we call these subgroups OCC 2 a $\left(195^{\circ}<l<15^{\circ}\right)$ and OCC $2 \mathrm{~b}\left(15^{\circ}<l<195^{\circ}\right)$. Comparing the visible distribution of the OCC 2 members with the extinction maps, one can find an agreement in the locations of the empty region and the Ophiuchus and Perseus-Auriga clouds. Thus, we cannot exclude that some OCC 2 members are possibly hidden by obscuring clouds.

Although less prominent, OCC $2 \mathrm{a}$ and OCC $2 \mathrm{~b}$ differ also in their location in the $Y Z$ plane. OCC $2 b$ shows a dispersed distribution and tends to a mean location below the Galactic plane, whereas the majority of the OCC 2 a members are above the Galactic plane. In the VPD of tangential velocities, the OCC $2 b$ members are distributed more compactly around the centre than the members of the OCC 2 a subgroup.

In Table 4 we list all OCC 2 candidates i.e., all T3 clusters located within $d_{x y}=0.65 \mathrm{kpc}$, which are not $1 \sigma$ members of the Perseus-Auriga group. For each cluster, the table contains the COCD number, the cluster designation, $(X, Y, Z)$ coordinates, the cluster age, and membership probability based on the distribution of tangential velocities in the VPD. The OCC $2 b$ candidates are marked by asterisks.

For eight $1 \sigma$ members of OCC 2 radial velocities are known, so we can determine the space velocity vector though, less reliably than for OCC 1 (see Table 7). According to the rotation velocity around the Galactic centre, OCC 2 outruns the open cluster centroid with a velocity $\bar{V}=3.4 \pm 1.2 \mathrm{~km} \mathrm{~s}^{-1}$. The average tangential velocity in longitude $\overline{T_{l}}=4.0 \pm 0.8 \mathrm{~km} \mathrm{~s}^{-1}$ indicates a significant rotation of OCC 2 with respect to the centroid of open clusters. With an average heliocentric distance of OCC 2 of about $0.5 \mathrm{kpc}$, we find an angular rotation velocity $\omega=8 \pm 1.6 \mathrm{~km} \mathrm{~s}^{-1} \mathrm{kpc}^{-1}$.

Summarising the main attributes of OCC 2, we conclude that the OCC 2 members show a number of properties expected for a real physical system. The complex is limited in space and it shows a significant (by a factor of 2) density enhancement over the surrounding background. The T3 sample has structural parameters $\left(Z_{0}\right.$ and $h_{Z}$ in Table 1$)$ which differ from the
Table 4. Candidates of the OCC 2.

\begin{tabular}{|c|c|c|c|c|c|c|}
\hline COCD & Name & $\begin{array}{r}X \\
\mathrm{kpc}\end{array}$ & $\begin{array}{r}Y \\
\mathrm{kpc}\end{array}$ & $\begin{array}{r}Z \\
\mathrm{kpc}\end{array}$ & $\begin{array}{r}\log t \\
\mathrm{yr}\end{array}$ & $\begin{array}{l}P_{\mathrm{t}} \\
\%\end{array}$ \\
\hline 3 & *Blanco 1 & 0.050 & 0.013 & -0.264 & 8.32 & 2 \\
\hline 15 & *Platais 2 & -0.107 & 0.136 & -0.102 & 8.54 & 100 \\
\hline 36 & *NGC 1039 & -0.387 & 0.285 & -0.134 & 8.42 & 4 \\
\hline 89 & NGC 2184 & -0.537 & -0.328 & -0.117 & 8.37 & 1 \\
\hline 96 & Collinder 95 & -0.516 & -0.206 & 0.000 & 8.36 & 100 \\
\hline 97 & Collinder 97 & -0.452 & -0.214 & -0.015 & 8.32 & 8 \\
\hline 141 & NGC 2396 & -0.397 & -0.433 & 0.027 & 8.52 & 100 \\
\hline 144 & NGC 2413 & -0.287 & -0.333 & 0.024 & 8.46 & 100 \\
\hline 151 & Ruprecht 27 & -0.266 & -0.492 & -0.025 & 8.41 & 100 \\
\hline 161 & Ruprecht 31 & -0.163 & -0.449 & -0.050 & 8.56 & 100 \\
\hline 168 & ESO 123-26 & 0.029 & -0.527 & -0.154 & 8.33 & 63 \\
\hline 200 & Ruprecht 65 & -0.060 & -0.496 & -0.014 & 8.57 & 100 \\
\hline 223 & Turner 5 & -0.040 & -0.391 & 0.077 & 8.49 & 0 \\
\hline 241 & NGC 3228 & 0.092 & -0.489 & 0.040 & 8.43 & 19 \\
\hline 245 & Loden 143 & 0.158 & -0.579 & -0.009 & 8.45 & 59 \\
\hline 246 & Loden 89 & 0.094 & -0.368 & 0.006 & 8.47 & 100 \\
\hline 247 & Loden 59 & 0.147 & -0.632 & 0.037 & 8.45 & 61 \\
\hline 276 & NGC 3532 & 0.167 & -0.468 & 0.012 & 8.45 & 65 \\
\hline 333 & Loden 915 & 0.310 & -0.391 & 0.028 & 8.44 & 24 \\
\hline 340 & Loden 1171 & 0.332 & -0.372 & 0.029 & 8.45 & 27 \\
\hline 344 & Loden 1194 & 0.336 & -0.370 & 0.016 & 8.53 & 72 \\
\hline 349 & ESO 175-06 & 0.386 & -0.390 & 0.038 & 8.60 & 63 \\
\hline 373 & NGC 6124 & 0.441 & -0.154 & 0.049 & 8.34 & 100 \\
\hline 392 & NGC 6281 & 0.482 & -0.105 & 0.017 & 8.51 & 100 \\
\hline 410 & Alessi 9 & 0.185 & -0.053 & -0.031 & 8.42 & 1 \\
\hline 419 & NGC 6469 & 0.546 & 0.063 & 0.019 & 8.36 & 86 \\
\hline 422 & NGC 6494 & 0.618 & 0.108 & 0.031 & 8.52 & 100 \\
\hline 463 & *Stock 1 & 0.174 & 0.304 & 0.014 & 8.53 & 14 \\
\hline 469 & *NGC 6828 & 0.405 & 0.432 & -0.097 & 8.56 & 41 \\
\hline 489 & *Roslund 6 & 0.093 & 0.440 & 0.002 & 8.47 & 7 \\
\hline 496 & *NGC 7058 & -0.020 & 0.400 & 0.004 & 8.35 & 21 \\
\hline 499 & *NGC 7092 & -0.013 & 0.310 & -0.012 & 8.57 & 0 \\
\hline 514 & *Stock 12 & -0.145 & 0.368 & -0.059 & 8.45 & 100 \\
\hline 1034 & ASCC 34 & -0.474 & -0.270 & 0.067 & 8.55 & 100 \\
\hline 1038 & ASCC 38 & -0.370 & -0.333 & 0.047 & 8.60 & 16 \\
\hline 1041 & ASCC 41 & -0.280 & -0.229 & 0.079 & 8.44 & 0 \\
\hline 1051 & ASCC 51 & 0.136 & -0.466 & -0.122 & 8.53 & 100 \\
\hline 1059 & ASCC 59 & 0.131 & -0.534 & -0.005 & 8.60 & 70 \\
\hline 1074 & ASCC 74 & 0.343 & -0.429 & 0.034 & 8.50 & 27 \\
\hline 1083 & ASCC 83 & 0.509 & -0.317 & 0.012 & 8.40 & 100 \\
\hline 1101 & *ASCC 101 & 0.128 & 0.318 & 0.070 & 8.52 & 100 \\
\hline 1106 & ASCC 106 & 0.374 & 0.318 & -0.093 & 8.42 & 1 \\
\hline 1109 & *ASCC 109 & 0.151 & 0.423 & 0.028 & 8.31 & 100 \\
\hline 1115 & *ASCC 115 & -0.078 & 0.594 & -0.027 & 8.59 & 100 \\
\hline 1123 & *ASCC 123 & -0.064 & 0.241 & -0.017 & 8.41 & 100 \\
\hline 1124 & *ASCC 124 & -0.119 & 0.576 & -0.119 & 8.48 & 100 \\
\hline
\end{tabular}


Table 5. Candidates of the Perseus-Auriga group of open clusters.

\begin{tabular}{rlrrrr}
\hline \hline COCD & Name & $\begin{array}{r}X \\
\mathrm{kpc}\end{array}$ & $\begin{array}{r}Y \\
\mathrm{kpc}\end{array}$ & $\begin{array}{r}\log t \\
\mathrm{kpc}\end{array}$ \\
\hline 37 & NGC 1027 & -0.553 & 0.539 & 0.021 & 8.55 \\
40 & Trumpler 3 & -0.333 & 0.300 & 0.035 & 8.34 \\
43 & King 6 & -0.361 & 0.269 & -0.001 & 8.41 \\
52 & NGC 1582 & -0.560 & 0.212 & -0.032 & 8.60 \\
55 & Alessi 2 & -0.441 & 0.232 & 0.056 & 8.60 \\
81 & Stock 10 & -0.375 & 0.055 & 0.023 & 8.42 \\
1012 & ASCC 12 & -0.478 & 0.146 & -0.016 & 8.42 \\
1023 & ASCC 23 & -0.567 & 0.126 & -0.149 & 8.45 \\
\hline
\end{tabular}

other samples of clusters, and for the OCC 2 members these parameters are even different from that of the T3 field clusters. The OCC 2 members have similar ages and they show similar (and relatively compact) kinematics. Therefore, we believe that OCC 2 with its $271 \sigma$-members represents a real group of clusters formed about $300 \mathrm{Myr}$ ago.

The other group of T3 clusters, which we call the PerseusAuriga group due to its special location in space is clearly separated in Figs. 6 and 8. The group has very compact kinematics, the average motion being $\left(\bar{T}_{1}, \bar{T}_{b}\right)=(-12.6 \pm$ $0.8,5.9 \pm 1.2) \mathrm{km} \mathrm{s}^{-1}$ with standard deviation of $(2.4 \pm 0.6,3.5 \pm$ $0.8) \mathrm{km} \mathrm{s}^{-1}$. This velocity dispersion is even smaller than the cosmic dispersion predicted for clusters of corresponding ages by Eq. (10) and shown in Fig. 6. However, the compactness of the velocity distribution is not surprising for the PerseusAuriga group. The velocity ellipses derived by Eq. (10) define a measure of cosmic dispersion for clusters of a given age, provided that the clusters are distributed all over the sky. Due to the definition of tangential velocities, their dispersion depends on galactic longitude. For a sample of clusters located within a small range of galactic longitudes (like the Perseus-Auriga group), the $T_{l}$-distribution should be more compact than predicted by Eq. (10).

Despite of attempts to find more members of the PerseusAuriga group outside the density excess, we could add only one more cluster. Thus, the group remains very compact in 3D-space. However, this does not exclude the possibility that there could be more members since the group is located behind the Perseus-Auriga complex of molecular clouds, and we see the its members in transparency windows within the clouds.

Only for two clusters of the Perseus-Auriga group radial velocities are available. Therefore, the accuracy of the space velocity is very poor. The data on the Perseus-Auriga group is presented in Tables 5 and 7. Since all clusters of the PerseusAuriga group have tangential velocities distributed within the corresponding dispersion ellipsoid, their kinematic probability is $100 \%$. Therefore, we exclude the probability column in Table 5.
Table 6. Candidates of the Hyades moving group of open clusters.

\begin{tabular}{rlrrrr}
\hline \hline COCD & Name & $\begin{array}{r}X \\
\mathrm{kpc}\end{array}$ & $\begin{array}{r}Y \\
\mathrm{kpc}\end{array}$ & $\begin{array}{r}\log t \\
\mathrm{kpc}\end{array}$ \\
\hline 13 & Alessi 1 & -0.427 & 0.651 & -0.184 & 8.85 \\
201 & Praesepe & -0.142 & -0.069 & 0.100 & 8.90 \\
459 & NGC 6738 & 0.500 & 0.489 & 0.038 & 9.16 \\
461 & NGC 6793 & 0.612 & 0.912 & 0.064 & 8.64 \\
462 & NGC 6800 & 0.510 & 0.857 & 0.069 & 8.59 \\
494 & NGC 6991 & 0.032 & 0.699 & 0.020 & 9.11 \\
502 & NGC 7209 & -0.118 & 1.152 & -0.150 & 8.65 \\
1099 & ASCC 99 & 0.267 & 0.076 & -0.039 & 8.71 \\
& Hyades & -0.045 & 0.000 & -0.019 & 8.90 \\
\hline
\end{tabular}

\subsection{The Hyades moving group of open clusters}

According to Fig. 1, the T4 sample does not show any peculiarity which would require a dedicated discussion. However, this sample, also, includes a cluster group which becomes evident due to its prominent motion detected in Sect. 4 (see Fig. 5).

We found six open clusters with considerably large tangential velocities. Only one of them, NGC 6991, has a radial velocity measured, so for this cluster we can determine directly the complete vector of space velocity corrected for the Galactic rotation which turns out to be $(U, V, W)=(-58,-4,2) \mathrm{km} \mathrm{s}^{-1}$. The other clusters are located at longitudes from $44^{\circ}$ to $124^{\circ}$ and therefore, their $-T_{l}$ velocities should more or less well coincide with $U$ component, which we have estimated to be between -30 and $-50 \mathrm{~km} \mathrm{~s}^{-1}$. All these clusters are relatively old, with $\log t$ between 8.57 and 9.16.

In order to explain a possible nature of their large tangential velocities, we considered distribution of the tangential velocities corrected for the Galactic rotation and for the Solar motion with respect to the cluster centroid versus longitude. Since these clusters have large space velocities, there is a good chance to observe a sine dependence described by Eq. (11) if the clusters belong to a common stream. Figure 9 shows this dependences for the T4 sample (panel c). For comparison, the distribution is also given for the samples T1 (panel a) and T3 (panel b) where the members of the complexes OCC 1, OCC 2 and the Perseus-Auriga group are marked, too.

The visible distribution of tangential velocities of these clusters can be fitted by a sine function. This indicates a possible stream of several clusters moving in the direction of the Galactic anticentre with a velocity of about $40 \mathrm{~km} \mathrm{~s}^{-1}$ with respect to the general cluster centroid. We found three other old clusters with large $U$-motions which fit to the sine function quite well though their tangential velocities are small (due to their close location to the centre - anticentre direction). Two of them are well known clusters, the Hyades and Praesepe, with an age of $\log t=8.9$ (both) and $U$-components relative to the cluster centroid of -34.1 and $-30.9 \mathrm{~km} \mathrm{~s}^{-1}$, respectively. It is possible (but not necessary) that these nine clusters form a common kinematic stream. The peculiarity of the spatial distribution of the clusters prevents us from applying the member selection techniques used for other groups. For a clear proof 
Table 7. Parameters of newly identified cluster complexes and groups.

\begin{tabular}{lccccccccccc}
\hline \hline $\begin{array}{l}\text { Complex, } \\
\text { group }\end{array}$ & $N$ & $\begin{array}{c}\log t \\
\mathrm{yr}\end{array}$ & \multicolumn{1}{c}{$\begin{array}{c}X_{\mathrm{c}} \\
\mathrm{kpc}\end{array}$} & \multicolumn{1}{c}{$\begin{array}{c}Y_{\mathrm{c}} \\
\mathrm{kpc}\end{array}$} & $\begin{array}{c}\text { size } \\
\mathrm{kpc}\end{array}$ & $\begin{array}{c}\bar{T}_{1} \\
\mathrm{~km} \mathrm{~s}^{-1}\end{array}$ & $\begin{array}{c}\bar{T}_{b} \\
\mathrm{~km} \mathrm{~s}^{-1}\end{array}$ & $\begin{array}{c}\bar{U} \\
\mathrm{~km} \mathrm{~s}^{-1}\end{array}$ & $\begin{array}{c}\bar{V} \\
\mathrm{~km} \mathrm{~s}^{-1}\end{array}$ & $\begin{array}{c}\bar{W} \\
\mathrm{~km} \mathrm{~s}^{-1}\end{array}$ & $\begin{array}{c}n \\
\text { OCC 1 }\end{array}$ \\
\hline OCC 2 & 23 & $6.67-7.88$ & -0.078 & -0.053 & $0.385,0.475$ & $-3.6 \pm 1.4$ & $+1.0 \pm 1.0$ & $-4.0 \pm 1.2$ & $-0.2 \pm 1.1$ & $+0.3 \pm 0.6$ & 23 \\
Perseus-Auriga & 27 & $8.48 ; 0.09$ & 0.000 & 0.000 & $0.325,0.65$ & $+4.0 \pm 0.8$ & $+2.9 \pm 0.7$ & $+1.9 \pm 4.2$ & $+3.3 \pm 1.2$ & $+2.5 \pm 1.3$ & 8 \\
Hyades $^{1}$ & 9 & $8.47 ; 0.09$ & -0.450 & 0.300 & 0.26 & $-12.6 \pm 0.8$ & $+5.9 \pm 1.2$ & $+4.6 \pm 13.6$ & $+21.1 \pm 9.3$ & $+4.3 \pm 4.3$ & 2 \\
\hline
\end{tabular}

${ }^{1}$ The mean tangential velocities were computed with 6 clusters (without the Hyades, Praesepe, and ASCC 99).

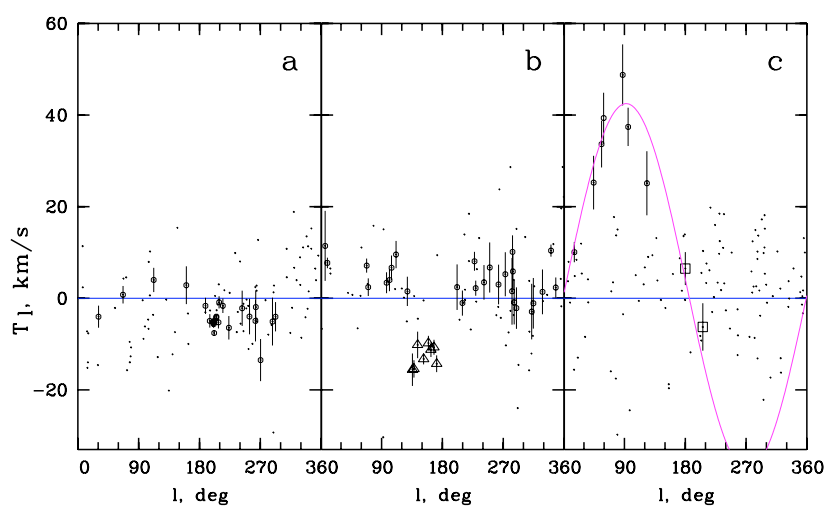

Fig. 9. Tangential velocities of clusters versus Galactic longitude. The velocities are corrected for differential Galactic rotation and for Solar motion with respect to the cluster centroid. The points mark clusters of the corresponding sample. Panel a) the T1 sample, circles are for the OCC 1 members. Panel b) the T3 sample, circles and triangles indicate OCC 2 and Perseus-Auriga members, respectively. Panel c) the T4 sample with candidates (circles) of a moving group. The Hyades and Praesepe are marked by squares. The distribution of tangential velocities of candidates is fitted by a sine function.

radial velocities would be essential. Therefore, we introduce this moving group only as a working hypothesis to explain the inhomogeneity in peculiar motion of clusters and call it the Hyades moving group after the most famous potential member. This cluster group must not be confused with the Hyades supercluster proposed by Eggen $(1958,1975)$ and recently studied by Famaey et al. (2005) who considered several hundreds of stars in the Solar vicinity (up to $500 \mathrm{pc}$ ) which have similar space velocities as the Hyades. In our case, there are a few old open clusters which are located at distances up to about $1.2 \mathrm{kpc}$ from the Sun but have ages and $U$-velocities comparable with those of the Hyades.

The list of candidates of the Hyades moving group is given in Tables 6 and 7.

\subsection{Summarising cluster complexes}

We classify the complexes OCC 1 and OCC 2 as real physical formations, the identification of which is supported by a sufficient amount of data. However, it is possible that not all their members are completely revealed. For the other two (the Perseus-Auriga and specially, the Hyades group), only fragmentary data are available. These make the discussion and argumentation of their nature rather difficult. To make a difference in the identification status, we refer to them as the groups of clusters. In Table 7 we summarise the parameters of the cluster complexes and the groups of clusters found in this study. The first column gives the names introduced in this paper, $N$ is the number of probable members. The average age with the standard deviation is given in Col. 3. For OCC 1 which probably shows a real age spread, we provide the age span only. The next three columns give the spatial data, the coordinates of the centre $(X, Y)$ and the size of the area occupied by the members. In this column, we provide the semiaxes for OCC 1 as described above. For OCC 2 the two numbers stand for the radii of the outer and the inner borders of the area of density excess. For the Perseus-Auriga and Hyades groups, the sizes are the radii of areas containing the selected candidates. $\bar{T}_{1}$ and $\bar{T}_{b}$ are the mean tangential velocities corrected for differential Galactic rotation and Solar motion with respect to the cluster centroid. For OCC 1, OCC 2, and the Perseus-Auriga group they were used for the kinematic selection of members (see Sect. 5.1). For the Hyades group we computed $\bar{T}_{1}$ and $\bar{T}_{b}$ only with clusters close to $l=90^{\circ}\left(l=44-124^{\circ}\right)$ where $\overline{T_{l}} \approx-\bar{U}$. The columns $\bar{U}, \bar{V}$, and $\bar{W}$ give the components of space velocity corrected for differential Galactic rotation and Solar motion with respect to the cluster centroid, and $n$ is the number of clusters used for computing these velocity components. This number indicates, that only for OCC 1 the space velocity was computed with all identified complex members. In OCC 2, less than $30 \%$ of its members can contribute to the determination of the space velocity. For the other groups, these values are merely illustrative. Figure 10 shows relative location of the discussed cluster groups in the $X Y$ plane.

Due to a relative proximity to the Sun and due to the completeness of kinematic data, OCC 1 is the most prominent structure found in our sample. One of the important features of this complex is its clustering in 3D-space. OCC 1 represents a density enhancement which is about a factor three higher than the surrounding density of field T1 clusters ( 75 vs. 25 clusters per square kpc). It is clearly outlined in space and shows a shape which is usually associated with Gould's Belt. It is worth to note that up to now Gould's Belt is studied only in connection with field OB stars (Frogel \& Stothers 1977; Torra et al. 2000), local gas (see Pöppel 1997, for a detailed review), and local associations (Stothers \& Frogel 1974; de Zeeuw 1999; and Bobylev 2004), and there was no attempt to study it in relation with young open clusters in a systematic way. 


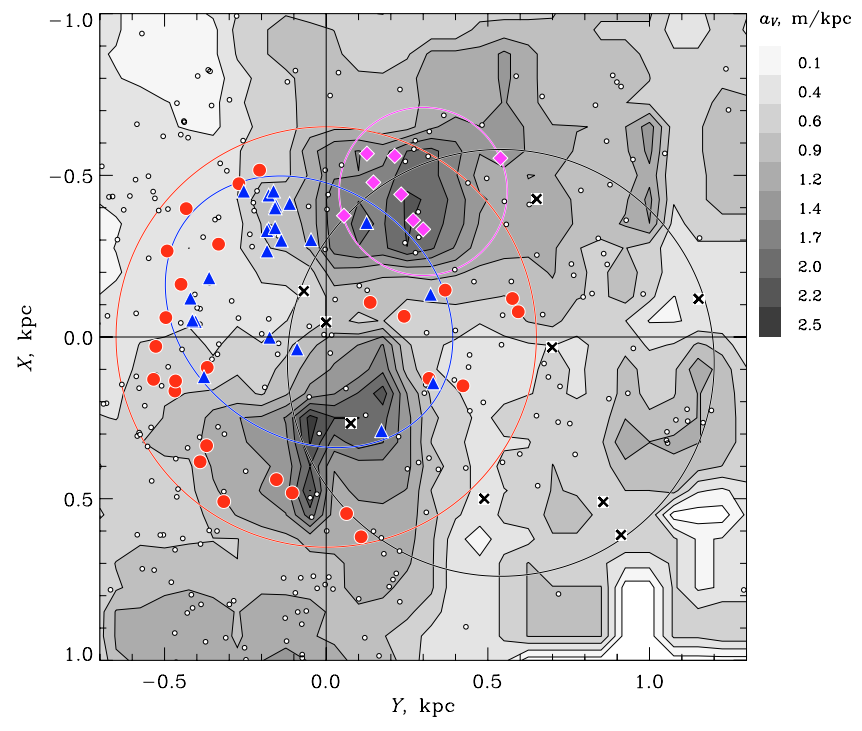

Fig. 10. The relative position of identified open cluster complexes and groups in the $X Y$ plane on a background of interstellar clouds revealed from data on our sample of clusters (Fig. 2). Large symbols mark candidate members for the Gould's Belt complex (triangles), OCC 2 (circles), the Perseus-Auriga group (diamonds), and the Hyades group (crosses). Small open circles mark field clusters. Large circles and the ellipse are apparent complex boundaries.

Based on the present data we can regard the Gould's Belt complex of open clusters as a physical system containing more than 20 clusters, occupying a flat elongated area of size of about $0.8 \times 1 \times 0.2 \mathrm{kpc}$. The cluster ages range from a few to about $80 \mathrm{Myr}$. About half of the clusters are concentrated in a prominent group residing in the region of the Orion association. They have the youngest ages (less the $25 \mathrm{Myr}$ ) among the OCC 1 clusters. The other clusters are distributed along the complex more uniformly, and as a rule, they are older than 25 Myr. From their location, we find them at the periphery of local molecular clouds with embedded associations which expand from Vela to Perseus via Scorpio-Centaurus. Only at the top of the spatial distribution above the Galactic plane (in the region of the IC 4665 and Stephenson 1 clusters), OCC 1 emerges out of the molecular clouds. The complex shows kinematics typical for young clusters, though it is more compact in the VPD than field clusters.

The first hint on possible cluster complexes was made by Lynga (1982), who identified three areas of high concentration of young $(\log t<7.5)$ clusters in the Perseus, Carina, and Sagittarius regions. Based on comparative statistics of populations of the suggested complexes and of the field, Janes et al. (1988) found that the three complexes contain mainly clusters younger than $40 \mathrm{Myr}$, and cover areas of order of 1.2...1.6 kpc. An extended search for clustering was undertaken by Efremov \& Sitnik (1988). Among stellar associations and young clusters (earlier than b2 in the scale of Becker \& Fenkart 1971), they identify 17 groups of various sizes $(150 \ldots 700 \mathrm{pc})$ containing at least 3 objects. They suggested that complexes of young clusters both outline the spiral arms and fill inter-arm space in the Solar neighbourhood (see Efremov 1995, for more details).
The presence of two cluster complexes and two more cluster groups in the Solar neighbourhood, with only one being regarded as a young object implies that such aggregates seem to be quite usual in the galactic disk. Therefore, it is not necessary to relate them to spiral arms or current star formation. If we do not suggest an exclusive role for the Solar neighbourhood, we may expect to find footprints of cluster groups (or their remnants) of different age everywhere in the Galactic disk. With time, such groups should loose their members due to cluster evaporation, become less prominent and will be detectable in the velocity space only.

We would like to stress that revealing the complexes and groups of clusters was not an output of a systematic search for such objects in the Galactic disk but rather the result of an attempt to understand the nature of irregularities either in density or in kinematic distribution which we found within the completeness area of the cluster sample. Therefore, one may expect that a systematic search in larger samples (which perhaps are less complete but cover larger areas) will result in identification of additional cluster agglomerations.

\section{Formation rate and lifetime of clusters}

One of the first extensive studies into the distribution of ages of open clusters and the estimation of their lifetime was carried out by Wielen (1971). The statistics of cluster ages was based on data from the catalogues of Lindoff (1968), and Becker \& Fenkart (1971). Later studies on this topic by Janes \& Adler (1982), Lyngå (1982), Pandey \& Mahra (1986), Pandey et al. (1987), Janes et al. (1988), Bhatt et al. (1989) and Battinelli \& Capuzzo-Dolcetta (1991) used the approach proposed by Wielen (1971), but considered the larger list of clusters by Janes \& Adler (1982) as well as the third, forth, and fifth versions of the Lund catalogue (Lyngå 1983, 1985, 1987). In comparison to these studies, our sample contains at least twice as many clusters with ages and distances determined by a uniform technique, and it includes many newly detected clusters. Therefore, a revision of the previous findings and conclusions is reasonable and relevant.

Let $\mathrm{d} N(t)=\psi(t) \mathrm{d} t$ be the number of clusters formed in some interval of time $(t, t+\mathrm{d} t)$, where $\psi(t)$ is the cluster formation rate (CFR). Further, we assume that the clusters decay with a probability $p=1 / \tau$ where $\tau$ is the typical lifetime of these clusters. If $N(a)$ is the number of clusters with age $a$, which are observed at the present time $t_{1}=t+a$, we can relate $N(a)$ to the CFR via the equation

$N(a)=N\left(t_{1}-a\right) \mathrm{e}^{-a / \tau}=\psi\left(t_{1}-a\right) \mathrm{e}^{-a / \tau}$.

If clusters would never dissolve (i.e., $\tau=\infty$ ), then their age distribution would reflect the temporal behaviour of the cluster formation rate. In contrast, for a constant CFR the age distribution $N(a)$ is controlled by the decay process. In the general case, the observed distribution of cluster ages depends on both factors, but in principle, it is possible to fix the processes. Since the observed span of cluster ages (a few Gyr) is much shorter than the age of the Galactic disk (over $10 \mathrm{Gyr}$ ) and since no indications of strong temporal variations are observed for the local rate of star formation (see e.g. Scalo 1986), 


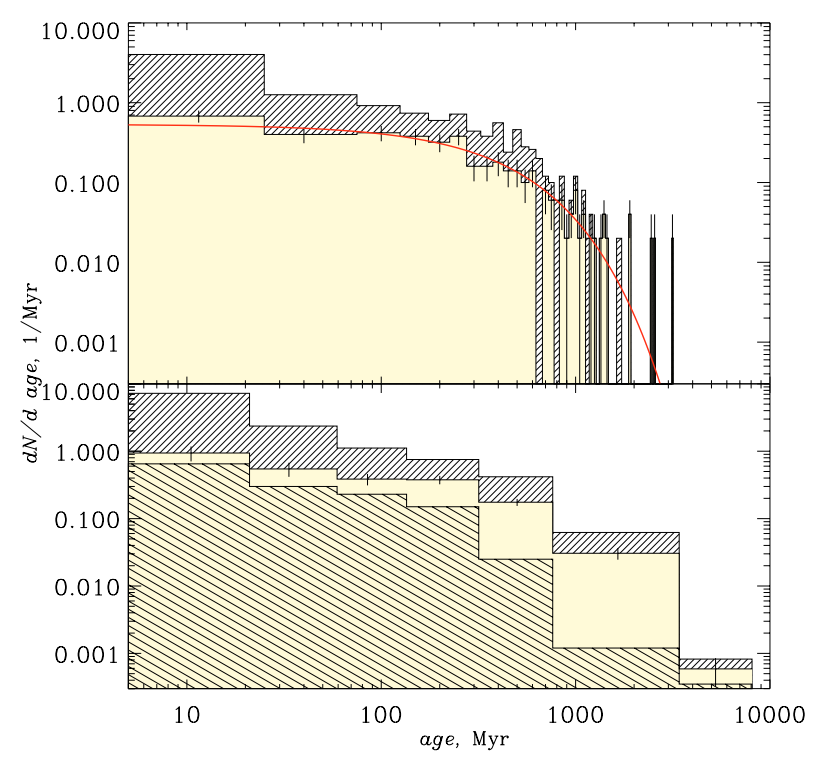

Fig. 11. Distribution of open clusters versus age. For an easier comparison, the distributions for the different samples are not normalised to unit area. Upper panel: our total sample is shown as the hatched histogram. The sample of field clusters within the completeness area is marked as the filled histogram, and the solid curve marks the fitted age distribution. The age step is $50 \mathrm{Myr}$. Lower panel: the same distributions as in the upper panel together with the age sample used by Wielen (1971). The data from Wielen (1971) are shown as the backhatched histogram in the foreground. The vertical bars correspond to Poissonian errors derived from cluster counts. The binning is chosen same as in Wielen (1971).

it is reasonable to assume that the formation rate of clusters is time-independent. Analysing the age distribution of open clusters under the assumption of a constant CFR during the disk lifetime, Wielen (1971) found a cluster halflifetime $t_{1 / 2}$ of $160 \mathrm{Myr}$, and a cluster formation rate of $\psi_{0}=$ $0.1 \mathrm{kpc}^{-2} \mathrm{Myr}^{-1}$. Here $t_{1 / 2}$ is the decay time of one half of the clusters once formed, and it can be derived from Eq. (13) as $t_{1 / 2}=-\tau \ln 1 / 2 \approx 0.7 \tau$. Using larger samples of clusters, the authors above obtained about the same or somewhat smaller lifetimes of open clusters. From a comparison of the age parameters for clusters of different richness classes and of different spatial location in the Galaxy, a reasonable conclusion had been achieved that the cluster lifetime increases with the richness class as well as outwards from the Galactic centre.

Taking into account the statistical properties of our cluster sample, we computed the CFR and the cluster lifetime for three cases. At first, we consider the total sample of 652 clusters. In the second case, we include only those 259 clusters which are located within the completeness area $\left(d_{x y} \leq 0.85 \mathrm{kpc}\right)$. Finally, in order to eliminate possible biases in the age distribution due to the cluster complexes, we excluded their $1 \sigma$-members from the second sample. This sample of so-called field clusters contains 195 clusters.

In the upper panel of Fig. 11 the distributions of clusters versus age are shown for the total sample as well as for the field clusters within the completeness area. As expected, the total sample is biased towards young clusters. Compared to the distribution of field clusters, this results in a steeper
Table 8. Cluster formation rates (CFR) and cluster lifetimes $(\tau)$.

\begin{tabular}{ccccll}
\hline \hline No & $\begin{array}{c}\text { CFR } \\
\mathrm{kpc}^{-2} \mathrm{Myr}^{-1}\end{array}$ & $\begin{array}{c}\tau \\
\mathrm{Myr}\end{array}$ & $N_{\mathrm{cl}}$ & Sample & Note \\
\hline 1 & $0.10 \pm 0.01$ & $256 \pm 12$ & 652 & tot & \\
2 & $0.31 \pm 0.03$ & $327 \pm 25$ & 259 & $\mathrm{cmp}$ & \\
3 & $0.23 \pm 0.03$ & $322 \pm 31$ & 195 & fld & \\
\hline 4 & 0.10 & 231 & 70 & $\mathrm{w} 71$ & 1 \\
5 & 0.18 & 144 & 112 & $\mathrm{p} 86$ & 2 \\
6 & 0.25 & 100 & 213 & $\mathrm{j} 88$ & 3 \\
7 & 0.45 & 14 & 100 & $\mathrm{~b} 91$ & 4 \\
\hline
\end{tabular}

Sample: No. 1...3 are the present study, No. 4 ...7 are taken from the literature; tot - the total sample; $\mathrm{cmp}$ - the sample of clusters within the completeness area; fld - the sample of field clusters within the completeness area; w71 - Wielen (1971); p86 - Pandey \& Mahra (1986); j88 - Janes et al. (1988); b91 - Battinelli \& Capuzzo-Dolcetta (1991). Notes: 1 - the Becker \& Fenkart (1971) sample; 2 - the Lund catalogue, 3rd edition; 3 - the Lund catalogue, 5th edition; 4 - clusters with $d \leq 2 \mathrm{kpc}$ and $V_{\text {integr }} \leq-4.5 \mathrm{mag}$.

average slope of the relation and, consequently, in smaller lifetimes. The curve obtained from fitting Eq. (13) coincides well with the observed distribution of field clusters. The parameters of the fit are given for all three cases in the first three rows of Table 8. We conclude that the completeness issue has a strong impact onto the determination of CFRs and cluster lifetimes. The exclusion of the complex members does not change the lifetime $\tau$ significantly, whereas the impact on $\psi$ is somewhat stronger: it decreases the CFR by about $20 \%$, but the difference is still within the mean errors.

In the bottom panel of Fig. 11 we show the same distributions together with results from Wielen (1971) based on the Becker \& Fenkart (1971) sample. In its shape, the distribution used by Wielen (1971) is in better agreement with the data of the total sample, but even so, there is a pronounced deficiency of older clusters which play an important role in the determination of cluster lifetime. This is the reason for the smaller lifetime derived by Wielen (1971), given in Table 8, too.

Table 8 also includes the corresponding findings by other authors from the $80 \mathrm{~s}$ and $90 \mathrm{~s}$. To ease comparison, we transformed $t_{1 / 2}$ to $\tau$, if the cluster lifetimes were not explicitly given in the original publication. Not only do the determinations of $\tau$ differ strongly among themselves, they are far off Wielen's results, and even farther from ours. We explain the disagreement of our results with the results of Pandey\& Mahra (1986), and Janes et al. (1988), based on the Lund Catalogue, by the considerable increase of data on clusters older than $250 \mathrm{Myr}$ in the last decade. For example, the number of clusters with ages of $250 \ldots 3000 \mathrm{Myr}$ within $0.85 \mathrm{kpc}$ from the Sun has been increased by a factor of 6 due to the newly discovered clusters. In comparison, the total number of clusters has grown from 1150 in the 5th edition of the Lund Catalogue to about 1700 clusters in Dias et al. (2004) i.e., by only a factor of 1.5. Concerning the extremely low lifetime $\tau$ derived by Battinelli \& Capuzzo-Dolcetta (1991, the selection effect is even stronger. Since they considered only the brightest clusters, their sample is overabundant on young objects, and the lifetime is strongly underestimated. 
The footprints of selection effects become also visible in the results for the CFR in Table 8 . Unlike the lifetime determination which mainly depends on a realistic relation between numbers of young and old cluster, the CFR results are more influenced by overall incompleteness of the sample studied. The increase of the CFRs computed with our samples correlates clearly with an increasing completeness of the data (cf. Table 8). A similar correlation can be observed for the CFR results derived by Wielen (1971), Pandey \& Mahra (1986), and Janes et al. (1988). The good agreement between our CFR and that from Janes et al. (1988) is expected, since we use the same basic source, the 5 th edition of the Lund Catalogue. On the other hand, we believe that the considerable disagreement in the CFR derived by Battinelli \& Capuzzo-Dolcetta (1991) and all others is a consequence of the underestimation of ages of their young clusters. It is well known that the turn-off age calibrations are biased in the case of young clusters (a few tens of Myr or younger) due to the rather steep Main Sequences of early-type stars towards younger ages. Neglecting this bias, one underestimates the age spread within a sample of young clusters, and hence, overestimate their abundance.

Taking the cluster lifetime derived above and an age $t_{1}$ for the Galactic disk of about 10-12 Gyr, we compute the total number of cluster generations $n_{\mathrm{g}}=t_{1} / \tau$ as being of the order of 30-40. Provided that a typical open cluster consists of $n_{\mathrm{o}}^{*}=1000$ stars at its birth and with the surface density of clusters being $\Sigma=114 \mathrm{kpc}^{-2}$ (see Sect. 3.3), the total surface density $n^{*}$ of disk stars and their remnants which have passed an open cluster member phase is $n_{\mathrm{g}} n_{\mathrm{o}}^{*} \Sigma \approx 4 \times 10^{6} \mathrm{kpc}^{-2}$. This number is in a good agreement with another estimate coming from the derived CFR i.e., $n^{*}=n_{\mathrm{o}}^{*} \psi t_{1} \approx 3.6 \times 10^{6}$. Compared with the local density of disk stars of about $7 \times 10^{7} \mathrm{kpc}^{-2}$, we find that less than $6 \%$ of the total stellar population of the Galactic disk is genetically connected with classical open clusters. On the other hand, this estimate is based on assumptions and extrapolations which at present can not be checked in detail. Therefore, we can only conclude that that portion of the disk stellar population, which had been originated in open clusters, is relatively small, of the order of $10 \%$.

This estimate is implicitly supported by another statistics available from the ASCC-2.5. Let us count all stars which are brighter than $V=9$ mag i.e., a magnitude range where $99 \%$ of stars have spectral classification and, practically, where all clusters are identified in the ASCC-2.5. Among the intrinsically bright population (supergiants, $\mathrm{O}-\mathrm{B} 3$ stars), we then computed the ratio of $1 \sigma$ and $2 \sigma$ cluster members to field stars. It turns out that about $10 \%$ of these 3770 extremely luminous stars are members of open clusters. Our results support the "pessimistic" estimate of $10 \%$ obtained by Miller \& Scalo (1978) from a much poorer statistics.

The rather low input of open clusters to the stellar population of the Galactic disk can be explained by their high infant mortality, reducing generations of newly born clusters by a half (Lamers et al. 2005). Even so, this is not sufficient to account for all Population I stars. Thus, the current statistics support the early conclusion by Miller \& Scalo (1978) that about $65 \%$ of the disk stars should have their origins in the association reservoir.

\section{Conclusions}

In this paper we studied unbiased properties of the local cluster population taking advantage of a homogeneous set of open cluster parameters derived from the all-sky census of cluster stars up to $V=12.5$. The revised cluster membership, the implementation of accurate kinematic and photometric data supplemented by radial velocities and spectral classification available at the moment, allowed us to extend the completeness area of open cluster data in the optical up to a distance of $0.85 \mathrm{kpc}$. The resulting sample was the basis for the determination of spatial, kinematical and evolutionary parameters of cluster population in the local Galactic disc.

Although the spatial distribution of clusters on scales comparable to inter-arm distances $\left(d_{x y} \approx 2 \mathrm{kpc}\right)$ is compatible with a model of a constant surface density, the distribution is not uniform. There are two main factors influencing the observed distribution in the optical. At first, the cluster can form groups which are revealed in the age distribution as well as in the space and velocity distributions. We found three cluster complexes in the Solar neighbourhood affecting significantly the apparent density pattern. The other effect influencing the apparent distribution of nearby open clusters in the optical is a patchy interstellar extinction due to local screening clouds. Also, these have a considerable impact on the observed distribution of clusters at large distances which we can see only through transparency windows. It is not excluded that some features of the local disk (like the Local arm) which have been discussed for a long time, are only virtual details which appear due to an uneven distribution of the reddening. The vertical distribution of the cluster sample turned out to be extremely flat, with a scale height of about $50 \ldots 60 \mathrm{pc}$, and it does not depend on age at least for cluster ages up to $1 \mathrm{Gyr}$. We found the symmetry plane of open clusters to be $Z_{0}=-22 \pm 4 \mathrm{pc}$, and the total density of clusters in the symmetry plane is $D\left(Z_{0}\right)=1015 \mathrm{kpc}^{-3}$. The spatial distribution of younger clusters is compatible with a flat grand design of a spiral pattern with a pitch angle of $p=-5^{\circ} \ldots-6^{\circ}$.

The kinematical parameters of the cluster sample are comparable to those of young field stars in the Solar neighbourhood though, the clusters cover distances which are typically larger by a factor of two than Hipparcos-based samples of field stars. This confirms that, in general, the observed cluster population is young. A combination of accurate cluster ages and kinematical parameters provides a possibility to study the temporal variation of the cosmic velocity dispersion. We found that in average, the dispersion of each velocity component increases by a factor of two during a period of $3 \mathrm{Gyr}$. The local velocity field within about $1.5 \mathrm{kpc}$ can be sufficiently well described by the linear model of differential rotation. But similar to the density distribution, the regular velocity pattern can be broken by groups of clusters with significantly different motions. So, we found a group of nine older clusters showing a large velocity component in the Galactic anti-centre direction.

Fluctuations in the spatial and velocity distributions can be attributed to the existence of three open cluster complexes containing up to a few tens of clusters of similar ages. The youngest complex, OCC $1(\log t<7.9)$, is a structure of about 
$1 \mathrm{kpc}$ in size inclined to the Galactic plane by $19^{\circ}$. Highly probable, it is a signature of Gould's Belt which has generally been considered in the literature to be an aggregation of the youngest field stars, gas and associations. The most populated complex, OCC 2, includes clusters of moderate age $(\log t \approx 8.45)$. The clusters of the Perseus-Auriga group have the same age but show different and compact kinematics. They are also very compact in their location and seen in voids of the Perseus-Auriga clouds. Possibly, we see only a part of a larger structure covered by clouds. The oldest $(\log t \approx 8.85)$ and the most loose group was detected due to a large peculiar motion (i.e., velocities corrected for Galactic rotation and Solar motion) of about $25-45 \mathrm{~km} \mathrm{~s}^{-1}$ in the Galactic anticentre direction. We note that the Hyades and Praesepe have about the same age $(\log t=8.9)$ and they have an $U$ velocity component of -34.1 and $-30.9 \mathrm{~km} \mathrm{~s}^{-1}$, respectively. Nevertheless, the real existence of the Hyades moving group of open clusters cannot be proven due to the lack of radial velocities of the suggested candidates.

From the parameters of spatial distribution, we estimated a total number of $10^{5}$ open clusters currently in the Galactic disk. Cluster lifetime and formation rate obtained from the age distribution of field clusters within the completeness area are found to be $322 \pm 31 \mathrm{Myr}$ and $0.23 \pm 0.03 \mathrm{kpc}^{-2} \mathrm{Myr}^{-1}$, respectively. Assuming a typical open cluster of the Pleiades type, one derives the total surface density of disk stars passed through the phase of open cluster members to be about $4 \times$ $10^{6} \mathrm{kpc}^{-2}$. Compared to the local density of disk stars of about $7 \times 10^{7} \mathrm{kpc}^{-2}$, one obtains that the input of open clusters into the total population of the Galactic disk is about $6 \%$.

Acknowledgements. This work was supported by DFG grant 436 RUS 113/757/0-1, RFBR grant 03-02-04028. We acknowledge the use of the Simbad database and the VizieR Catalogue Service operated at the CDS, and the WEBDA facility at Observatoire de Geneva. We are indebted to A. V. Loktin for making his data available prior to publication, and V. S. Avedisova for useful discussions. We are grateful to the anonymous referee for his/her useful comments.

\section{References}

Alessi, B. S., Moitinho, A., \& Dias, W. S. 2003, A\&A, 410, 565

Arenou, F., Grenon, M., \& Gómez, A. 1992, A\&A, 258, 104

Bahcall, J. N., \& Soneira, R. M. 1980, ApJS, 44, 73

Battinelli, P., \& Capuzzo-Dolcetta, R. 1991, MNRAS, 249, 76

Becker, W. 1963, ZfA, 57, 117

Becker, W., \& Fenkart, R. 1971, A\&AS, 4, 241

Bhatt, B. C., Pandey, A. K., \& Mahra, H. S. 1989, Bull. Astr. Soc. India, 17, 35

Bica, E., Dutra, C. M., \& Barbuy, B. 2003a, A\&A, 397, 177

Bica, E., Dutra, C. M., Soares, J., \& Barbuy, B. 2003b, A\&A, 404, 223

Bobylev, V. V. 2004, Astr. Lett, 30, 785

Branham, L. R., Jr. 2002, ApJ, 570, 190

Dame, T. M., Hartmann, D., \& Taddeus, P. 2001, ApJ, 547, 792

Danilov, V. M., \& Seleznev, A. F. 1993, Astron. Astrophys. Trans., 6, 293

Dehnen, W., \& Binney, J. J. 1998, MNRAS, 298, 387

Dias, W. S., Alessi, B. S., Moitinho, A., \& Lépine, J. R. D. 2002, A\&A, 389, 871
Dias, W. S., Lépine, J. R. D., Alessi, B. S., \& Moitinho, A. 2004, Open clusters and Galactic structure, Version 2.0, http://www.astro.iag.usp.br/ wilton

Dutra, C. M., Bica, E., Soares, J., \& Barbuy, B. 2003, A\&A, 400, 533 Efremov, Yu. N. 1995, AJ, 110, 2757

Efremov, Yu. N., \& Sitnik, T. G. 1988, Sov. Astr. Lett., 14, 347

Eggen, O. J. 1958, MNRAS, 118, 65

Eggen, O. J. 1975, PASP, 87, 37

Famaey, B., Jorisse, A., Luri, X., et al. 2005, A\&A, 430, 165

Feast, M. W., \& Whitelock, P. 1997, MNRAS, 291, 683

Frogel, J. A., \& Stothers, R. 1977, AJ, 82, 890

FitzGerald, M. P. 1968, AJ, 73, 983

Janes, K. A., \& Adler, D. 1982, ApJS, 49, 425

Janes, K. A., Tilley, C., \& Lyngå, G. 1988, AJ, 95, 771

Joshi, Y. C. 2005, MNRAS, 362, 1259

Kharchenko, N. V. 2001, Kinematics and Physics of Celestial Bodies 17, 409 (ASCC-2.5, Cat. I/280A)

Kharchenko, N. V., Piskunov, A. E., \& Scholz, R.-D. 2004a, Astron. Nachr., 325, 439

Kharchenko, N. V., Piskunov, A. E., Röser, S., Schilbach, E., \& Scholz, R.-D. 2004b, Astron. Nachr., 325, 740 (Paper I)

Kharchenko, N. V., Piskunov, A. E., Röser, S., Schilbach, E., \& Scholz, R.-D. 2005a, A\&A, 438, 1163 (Paper II)

Kharchenko, N. V., Piskunov, A. E., Röser, S., Schilbach, E., \& Scholz, R.-D. 2005b, A\&A, 440, 403 (Paper III)

Lallement, R., Welsh, B. Y., Vergely, J. L., Crifo, F., \& Sfeir, D. 2003, A\&A, 411, 447

Lamers, H. J. G. L. M., Gieles, M., Bastian, N., et al. 2005, A\&A, 441, 117

Lindoff, U. 1968, Arkiv. Astr., 5, 1

Loktin, A. V. 2004, private communication

Loktin, A. V., Gerasimenko, T. P., \& Malysheva, L. K. 2001, Astron. Astrophys. Trans., 20, 607

Lucke, P. B. 1978, A\&A, 64, 367

Lyngå, G. 1982, A\&A, 109, 213

Lyngå, G. 1983, Catalogue of open clusters data, Third edition, CDS, Strasbourg (VII/41)

Lyngå, G. 1985, Catalogue of open clusters data, Forth edition, CDS, Strasbourg (VII/66)

Lyngå, G. 1987, Catalogue of open clusters data, Fifth edition, CDS, Strasbourg (VII/92)

Miller, G. E., \& Scalo, J. M. 1978, PASP, 90, 506

Neckel, Th., \& Klare, G. 1980, A\&AS, 42, 251

Olano, C. A. 1982, A\&A, 112, 195

Pandey, A. K., \& Mahra, H. S. 1986, Ap\&SS, 126, 167

Pandey, A. K., Bhatt, B. C., \& Mahra, H. S. 1987, Ap\&SS, 129, 293

Platais, I., Kozhurina-Platais, V., \& van Leeuwen, F. 1998, AJ, 116, 2423

Pöppel, W. G. L. 1997, Fundam. Cosmic Phys., 18, 1

Reed, B. C. 2005, AJ, 130, 1652

Ruprecht, J., Balazs, B., \& White, R. E. 1981, Catalogue of Star Clusters and Associations, Supplement 1, Associations, Akademiai Kiado (Budapest: Publ. House Hungarian Acad. Sciences)

Saio, H., \& Yoshii, Y. 1979, PASP, 91, 553

Scalo, J. 1986, Fund. Cosmic Phys., 11, 1

Schlegel, D. J., Finkbeiner, D. P., \& Davis, M. 1998, ApJ, 500, 525

Sharov, A. S. 1963, AZh, 40, 900

Stothers, R., \& Frogel, J. A. 1974, AJ, 79, 456

Torra, J., Fernandez, D., \& Figueras, F. 2000, A\&A, 359, 82

Valée, J. P. 1995, ApJ, 454, 119

van Schewick, H. 1971, Veröff. Univ. Sternvarte Bonn, 84, 1

Wielen, R. 1971, A\&A, 13, 309

de Zeeuw, P. T., Hoogerwerf, R., de Bruijne, J. H. J., Brown, A. G. A., \& Blaauw, A. 1999, AJ, 117, 354 